\title{
Knudsen diffusivity in random billiards: spectrum, geometry, and computation
}

\author{
T. Chumley*, R. Feres, L. A. Garcia German ${ }^{\dagger}$
}

April 13, 2021

\begin{abstract}
We develop an analytical framework and numerical approach to obtain the coefficient of self-diffusivity for the transport of a rarefied gas in channels in the limit of large Knudsen number. This framework provides a method for determining the influence of channel surface microstructure on the value of diffusivity that is particularly effective when the microstructure exhibits relatively low roughness. This method is based on the observation that the Markov transition (scattering) operator determined by the microstructure, under the condition of weak surface scattering, has a universal form given, up to a multiplicative constant, by the classical Legendre differential operator. We also show how characteristic numbers of the system - namely geometric parameters of the microstructure, the spectral gap of a Markov operator, and the tangential momentum accommodation coefficient of a commonly used model of surface scattering - are all related. Examples of microstructures are investigated to illustrate the relation of these quantities numerically and analytically.
\end{abstract}

\section{INTRODUCTION}

A motivating question and our model. In the idealized experiment shown in Figure 1, a pulse of inert gas at low pressure is pumped into a long but finite tube, which we refer to as the channel. The inner surface of the channel has some degree of roughness due to its molecular structure and surface irregularities. The experimenter is able to measure the rate of gas outflow using some device such as a mass spectrometer, which generates data of the kind represented by the graph on the right-hand side of the figure. From such data, transport characteristics of the gas flow through the channel can be derived, as described in [7. We assume a sufficiently small pulse, under vacuum conditions, to insure that molecular mean free path is much larger than the diameter of the channel. Thus collisions between the gas molecules can be ignored

*Department of Mathematics and Statistics, Mount Holyoke College, 50 College St, South Hadley, MA 01075

${ }^{\dagger}$ Department of Mathematics and Statistics, Washington University, Campus Box 1146, St. Louis, MO 63130 
while gas-surface interaction is expected to influence transport properties most prominently. The property of interest here, which can be indirectly measured from such an experiment, is the Knudsen self-diffusivity coefficient of the gas, as explained, for example, in [7]. The central question we wish to address is: How do the surface characteristics affect the Knudsen self-diffusivity?

In this paper, we assume that gas-surface interaction amounts to perfectly elastic, or billiardlike, collisions between point masses (the gas molecules, also referred to here as particles) and the channel surface, and hence energy exchange between surface and molecules will be ignored. We assume moreover that the channel is two-dimensional and that its surface microstructure is static and periodic, and can be described by a relatively small number of geometric parameters. Thus the mathematical problem we pose here is to determine how the Knudsen self-diffusivity explicitly depends on these parameters.

In the large Knudsen number limit (i.e., for large mean free paths), molecular trajectories are independent of each other and the diffusion process is derived from an analysis of individual trajectories of particles undergoing a random flight inside the channel. This random flight is governed by a Markov operator $P$ that gives, at each particle-surface collision, the post-collision velocity of the particle as a random function of the pre-collision velocity. All the information about the periodic surface geometry relevant to the task of obtaining diffusivity is encoded in $P$. In fact, diffusivity corresponds to the variance of a one-dimensional Wiener process obtained from the random flight determined by $P$ via a Central Limit Theorem. (As explained in [7, this variance can be obtained from the mean exit time in the limit of long channel lengths in the context of the above idealized experiment. The mean exit time, as a function of the channel length, is the only information that needs to be extracted from the exit flow rate data. We won't deal here with this particular aspect of the analysis and assume, in effect, that the channel is infinite in length.) Our main goals are thus centered around two issues. First, we aim to establish a functional, analytic relationship among the aforementioned variance, the spectrum of the Markov operator $P$, and parameters of the geometric microstructure. Second, we aim to obtain effective numerical methods for finding this dependence for any given geometric microstructure.

Main results. The main results in the paper are centered around a detailed study of the Markov operator $P$ and establish analytic and probabilistic properties of $P$ and its corresponding Markov chain. To begin, we show that for a large class of microstructures, $P$ has a positive spectral gap, which in turn establishes the ergodicity of the Markov chain as well as the fact that functionals of the Markov chain satisfy the Central Limit Theorem. We have shown in previous work [11, 14] that $P$ is a self-adjoint, compact or quasi-compact operator on an appropriate Hilbert space, for microstructures whose sides are concave with curvature bounded away from zero. However, the present work establishes a positive spectral gap of $P$ for a significantly larger class of microstructures. Using a conditioning technique, we show that $P$ has positive spectral gap when only a certain positive measure portion of the billiard phase space is dispersing.

It is now a classical result in the theory of Markov chains [16] that one can obtain an expression of the diffusivity of the Markov chain corresponding to $P$ in terms of an integral over the spectrum of $P$. A key insight of the present work is that, for relatively flat microstructures, these quantities, namely the diffusivity and the spectral gap of $P$, are directly connected with a single summary geometric parameter that can be computed in a straightforward way from a description of the surface microstructure, which we call the surface flatness parameter and 


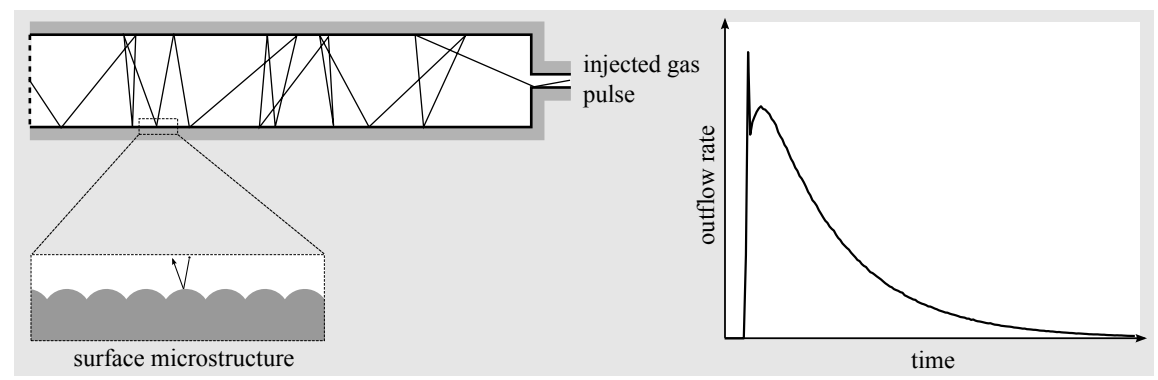

Figure 1: Idealized experiment for measuring diffusivity of a rarefied gas flow through a channel. In the limit of large mean free path, trajectories of gas molecules (point masses) injected into the channel as a short pulse, are independent of each other and their stochastic behavior provides information about the geometric microstructure of the inner surface of the channel. From exit flow rate data one determines the Knudsen self-diffusivity. The mathematical problem posed in this paper is the explicit determination of the diffusivity constant as a function of geometric parameters defining the microstructure.

denote by $h$. The connection between these three properties of the system is obtained based on a fact which, to the best of our knowledge, has not been noted previously in the context of computing the Knudsen diffusivity. When scattering by $P$ is relatively weak (in a sense to be made precise), it is natural to approximate the operator $P$ in the form $P=I+\mathcal{L}$, where $I$ is the identity operator and $\mathcal{L}$ may be expected to take the form of a differential (velocity diffusion) operator. We show that $\mathcal{L}$ has a universal form: it is a constant (namely, up to a factor of 2 , our flatness parameter $h$ ) times the Legendre operator, whose (purely discrete) spectrum is known explicitly. We are able to exploit the approximation of $P$ by the Legendre operator to give an asymptotic expression and error estimates for the Knudsen diffusivity in terms of $h$.

The conceptual link we obtain between $h$, the Knudsen diffusivity, and the spectral gap of $P$ is, in our opinion, a new theoretical insight in a very classical subject, which also yields a very effective method of computation, at least in the case of small values of $h$. The final concern of this work is to obtain and validate effective numerical methods for computing the Knudsen self-diffusivity in terms of the geometric microstructure parameters, in both the small and large $h$ case. This will be discussed in detail in Subsections 2.4 and 2.6 and Sections 4 and 5 . A number of numerical experiments involving different microstructures will also be explored.

Remarks on assumptions. We make a few remarks about the assumptions in our model. The analysis developed in this paper does not require in an essential way all the assumptions made, but we hope that the greater simplicity of the present set-up will help to make clearer the main points. For example, we have made a deliberate choice to consider periodic profiles of microstructures with relatively few geometric parameters to emphasize the relationship between geometric parameters, the spectrum of the Markov operator, which in turn establishes the relationship between geometric parameters and Knudsen diffusivity. While it's possible to give similar formulas relating Knudsen diffusivity and geometric parameters for, say, randomly chosen profiles, such formulas are straightforward but tedious to express, and we fear would muddle the main point.

When studying the Knudsen self-diffusivity, the observable which measures particle flight 
between collisions in the channel has infinite variance. A study of the Central Limit Theorem and Knudsen diffusivity for a different class of random billiard Markov chains with infinite variance observables has been done previously in [7]. While the methods in the present work can be adapted to the case of infinite variance observables, we have chosen to use a cut-off observable to reduce to the finite variance case for the sake of clarity. Besides clarity, there are a number of physically relevant reasons for considering the cut-off observable we have used in our examples. Namely, (1) the cut-off can arise for macroscopic curvature of the channel in which the test particle traverses. It can also arise due to (2) a finite mean free path resulting from unlikely but non-zero probability particle-particle collisions in the large but finite Knudsen number regime, and (3) real systems where the channel is of finite length and bounded on either end. These physically relevant mechanisms are discussed in detail in [4].

Finally, we should note that in the case of three dimensional cylinders, the inter-collision distance observable is always of finite variance, so our methods in the current paper serve as prototypes for this generalization. The techniques we introduce here are in fact not particular to dimension 2. Indeed, a multivariable Legendre operator on the unit disc, whose spectral theory is explicitly known, plays the same role in higher dimensions as the classical Legendre operator does in the present work. The details of this approximation and the corresponding models in higher dimensions - where we consider three-dimensional cylindrical channels, parallel plates, and allow for collisions that induce energy exchange between gas and surface at a given surface temperature - are at the core of future work currently in preparation.

Related work. Better understanding of rarefied gas transport has practical implications for a number of engineering fields including high altitude gas dynamics, porous media, vacuum technology, nano- and microfluidics, among others. These applications have stimulated much experimental work. The following list of papers is a far from thorough or systematic sample of such work: [1, 17, 18, 20, 23]. The reader interested in the more applied side of the subject should consult these sources and others cited in them. From a purely mathematical perspective, this is a rich source of well motivated and potentially fruitful problems in the general theory of stochastic processes, and, more specifically, in the study of the stochastic dynamics of random billiard systems. This is our main motivation for studying the subject. We mention from the mathematical literature the following, also necessarily incomplete, list: [3, 5, 8, 10, 15].

Organization of the paper. The rest of this paper is organized as follows. In Section 2 we detail our main results after introducing the necessary definitions; we define what we call the random billiard Markov chain model in detail and state some of its basic properties. Among the main results stated in Section 2 (and proved in more general form later in the paper) we have that under certain geometric conditions on the boundary microstructure, the Markov chain has positive spectral gap and is uniformly ergodic. Numerical evidence for this is then given for a few examples. With ergodicity in hand, we discuss the central limit theory of the Markov chain providing explicit expressions for the variance of the limit diffusion in terms of the Markov operator $P$. The main analytic technique for computing diffusivity, based on a Galerkin method for solving a Markov-Poisson equation and a key observation that $P$ is closely related to the Legendre differential operator, is also given in this introductory section. This approach for obtaining diffusivity is then compared with other more straightforward methods for a family of microstructures we call the simple bumps family. A few more examples of microstructures are explored, having in mind the relation between geometric parameters, diffusivity, and spectral gap. Section 3 is dedicated to stating and proving the analytical results of the paper in their 
general form, while Section 4 details, and adds further information, to the numerical methods and their validation.

\section{MAin DEFINITIONS AND RESUlts}

\subsection{The BILliard CELl AND its TRANSition Operator $P$}

The notation $\mathcal{P}(\Omega)$ will be used below to denote the space of probability measures on a measurable space $\Omega$. If $\mu$ is a measure on $\Omega$ and $f: \Omega \rightarrow \mathbb{R}$ is $\mu$-integrable, we write the integral of $f$ with respect to $\mu$ as

$$
\mu(f):=\int_{\Omega} f(\omega) \mu(d \omega) .
$$

The Hilbert space of square integrable functions with respect to $\mu$ and its subspace of functions with mean zero will be written

$$
L^{2}(\Omega, \mu)=\left\{f: \mu\left(f^{2}\right)<\infty\right\}, L_{0}^{2}(\Omega, \mu)=\left\{f \in L^{2}(\Omega, \mu): \mu(f)=0\right\},
$$

with inner product $\langle f, g\rangle_{\mu}:=\int_{\Omega} f(\omega) g(\omega) \mu(d \omega)$ and norm $\|f\|_{\mu}:=\langle f, f\rangle_{\mu}^{1 / 2}$. Moreover, we define a norm on the space of square integrable probability measures on $\Omega$ which are absolutely continuous with respect to $\mu$ as follows. Let $\nu$ be such a measure, so that $f$ is the Radon-Nikodym derivative of $\nu$ with respect to $\mu$. Then $\|\nu\|_{\mu}:=\|f\|_{\mu}$.

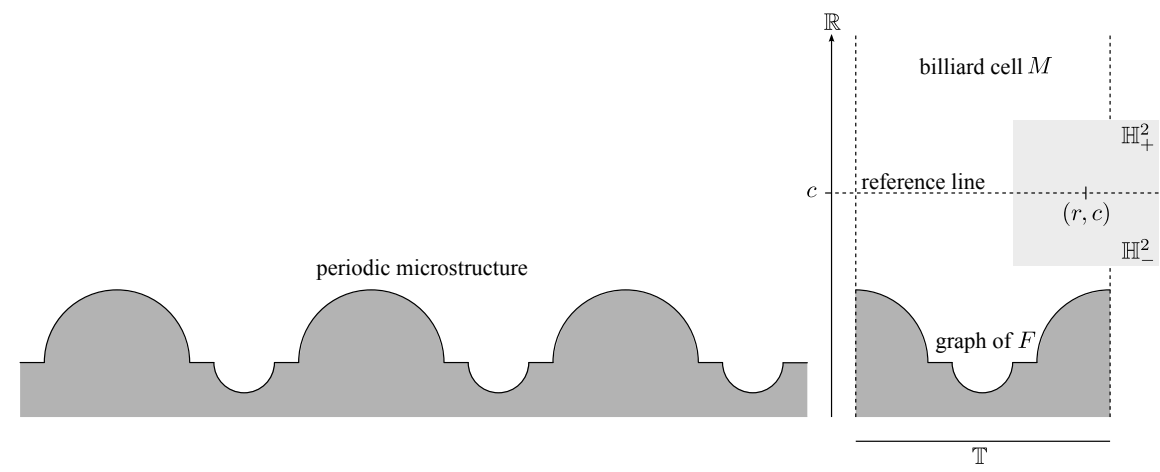

Figure 2: A periodic microstructure and its billiard cell, with some of the notation used to define the random billiard map and its transition operator $P$. For some of our results we assume that the boundary curve is the graph of a piecewise smooth function $F: \mathbb{T} \rightarrow \mathbb{R}$.

The general set-up will be that of a two-dimensional random billiard with static, periodic, geometric microstructure, as in 17, 9, 11, 12, 13, 14. The periodic structure is defined by the choice of a billiard cell $M$, from which the Markov operator $P$ will be defined. The billiard cell is a subset $M$ of $\mathbb{T} \times \mathbb{R}$, where $\mathbb{T}$ denotes the 1-dimensional torus (equivalently, the interval $(0, \ell)$ with periodic condition imposed at the endpoints, where $\ell$ will typically be set equal to 1 .) The boundary of the billiard cell is assumed to be a piecewise smooth curve. For some of the results given below, the boundary will be the graph of a piecewise smooth function $F: \mathbb{T} \rightarrow \mathbb{R}$, so that $M$ consists of the points $(r, y)$ such that $y \geq F(r)$. Choose an arbitrary value $c$ such that $c>F(r)$ 
for all $r \in \mathbb{T}$. The line $y=c$ will be called the reference line. At any point $(r, c)$ on the reference line we define the half spaces $\mathbb{H}_{-}^{2}$ and $\mathbb{H}_{+}^{2}$ of incoming and outgoing velocities, respectively. Thus $(r, c, v) \in M \times \mathbb{H}_{-}^{2}$ represents the initial conditions of an incoming particle trajectory. These conditions uniquely specify (for almost every $r$ and $v$ ) a billiard trajectory: upon hitting a non-corner point on the cell boundary, the particle reflects specularly without changing speed, and upon crossing a vertical boundary line of $M$ (more precisely, a line separating two adjacent cells, represented in Figure 2 by the vertical dashed lines) it reenters the other (dashed) line with unchanged velocity. With probability 1 on the set of initial conditions (due to Poincaré's recurrence), the trajectory returns to the reference line, at which point we register its outgoing velocity $V(r, v) \in \mathbb{H}_{+}^{2}$ and new position $r^{\prime}$. Without risk of confusion we may identify (through reflection about the reference line) $\mathbb{H}_{-}^{2}$ and $\mathbb{H}_{+}^{2}$, denoting both by $\mathbb{H}^{2}$. We have thus defined a transformation $(r, v) \mapsto\left(r^{\prime}, V(r, v)\right)$ (for almost all initial conditions $\left.(r, v)\right)$ on $\mathbb{T} \times \mathbb{H}^{2}$. We call this transformation the return billiard map.

Note that the vector norms satisfy $|v|=|V|$ since collisions are elastic. We may, without loss of generality, assume that the particle trajectories have unit speed. The incoming or outgoing state space, consisting of initial or return velocities, can then be taken to be the interval $X=(0, \pi)$ of angles the particle velocity makes with the reference line. We can (and often will) equivalently define $X=(-1,1)$ as the set of values of the cosine of those angles. Given an initial velocity $x \in \mathcal{X}$, we will often denote the return velocity by $X(r, x) \in \mathcal{X}$ in analogy with the earlier notation of velocities $v$ and $V(r, v)$ in $\mathbb{H}^{2}$.

Let $\mathcal{P}(X)$ denote the space of probability measures on $X$. Given an incoming velocity $v$, let us suppose that $r=U$ is a random variable with the uniform distribution over $\mathbb{T}$. Thus $X(U, x)$ becomes a random variable. We now define the Markov (or transition probabilities) operator $P$ as follows. Let $f$ be any bounded and continuous function on $X$ and define

$$
(P f)(x):=E[f(X(U, x))]=\int_{\mathbb{T}} f(X(r, x)) d r,
$$

where $d r$ is the length element of normalized Lebesgue measure on $\mathbb{T}$. Equivalently, we define a sequence of random variables $\left(X_{n}\right)_{n \geq 0}$ with a given initial distribution $\mu \in \mathcal{P}(X)$ as follows. Let $\left(U_{n}\right)_{n \geq 0}$ be an independent, identically distributed sequence of random variables uniformly distributed on $\mathbb{T}$, and, for each $n \geq 0$, let

$$
X_{n+1}:=X\left(U_{n}, X_{n}\right) .
$$

The justification for assuming, at each scattering event, that the point $r$ of entry over the opening of a billiard cell is random and uniformly distributed is due to our regarding the billiard cell as being very small relative to other length scales; any small uncertainty in the incoming velocity will make $r$ nearly fully uncertain. See [13, for a more detailed explanation of this point.

We can also regard $P$ as a map from $\mathcal{P}(X)$ to itself: Given any $\mu \in \mathcal{P}(X)$, let $\mu P \in \mathcal{P}(X)$ be such that for any test function $f$ (bounded and continuous),

$$
(\mu P)(f):=\mu(P f) .
$$

The following summarizes the basic properties of $P$. For their proofs, see [11, 14]. We say that the billiard cell $M$ is bilaterally symmetric (or simply symmetric) if it is invariant under reflection through the middle vertical line. When the boundary of the cell is the graph of a function $F$, this means that $F(r)=F(\ell-r)$ for all $r \in(0, \ell)$. 
Proposition 1. The Markov operator P, for any given billiard cell, has the following properties.

1. The measure $\pi \in \mathcal{P}(X)$ given by $\pi(d \theta)=1 / 2 \sin \theta d \theta$ is stationary of $P$. That is, $\pi P=\pi$.

2. As an operator on $L^{2}(X, \pi), P$ has norm 1 .

3. If $M$ is symmetric, $P$ is self-adjoint and the stationary Markov chain is reversible.

Note that when $X=(-1,1)$, it is straightforward to see by a change of variables that the stationary measure $\pi$ is given by the uniform measure $\pi(d x)=1 / 2 d x$.

If the the billiard cell is not bilaterally symmetric, the adjoint of $P$ is still closely related to $P$ as described in [9] and much of the analysis developed in this paper still applies. For simplicity, we do not consider the more general type of cells here.

\subsection{SPECTRAL GAP AND ERGODICITY}

Let $\left(X_{n}\right)_{n \geq 0}$ be the Markov chain with transition operator $P$ and initial distribution $\mu$. Then the measure $\mu P^{n}$ is the law of the $n$th step $X_{n}$. We are interested in the convergence of $\mu P^{n}$ to the stationary measure $\pi$ in the sense of total variation. Recall that the total variation of a measure $\mu$ is defined as

$$
\|\mu\|_{v}:=\sup _{A \subset X}|\mu(A)| .
$$

Definition 1. A Markov chain with stationary distribution $\pi$ is $\pi$-a.e. geometrically ergodic if there exists $0<\rho<1$ such that for $\pi$-a.e. $x \in X$ there exists a constant $M_{x}>0$ possibly dependent on $x$ such that $\left\|\delta_{x} P^{n}-\pi\right\|_{v} \leq M_{x} \rho^{n}$ for all $n \geq 1$.

The operator $P$ has spectral gap if there exists a constant $0<\rho<1$ such that

$$
\|P f\|_{\pi} \leq \rho\|f\|_{\pi}
$$

for all $f \in L_{0}^{2}(X, \pi)$. The value $\gamma:=1-\rho$ is called the spectral gap of $P$. It is straightforward to see that for a compact and self-adjoint $P, \rho$ is given by the largest eigenvalue of $P$ restricted to $L_{0}^{2}(X, \pi)$ and $\gamma>0$. Finally, we note that if $P$ has spectral gap and is self-adjoint, then for any initial distribution $\mu$ which is absolutely continuous with respect to $\pi$, there exists a constant $M_{\mu}>0$ such that

$$
\left\|\mu P^{n}-\pi\right\|_{v} \leq M_{\mu} \rho^{n} .
$$

See [19]. We will prove geometric ergodicity for a large class of microstructures satisfying certain geometric conditions.

The following is a special case of a more general result to be stated and proved in Section 3 . We call the height of the billiard cell the supremum of the $y$ coordinate function restricted to the boundary of the cell.

Theorem 1. Let $P$ be the Markov transition operator for a random billiard Markov chain whose billiard cell is symmetric and satisfies the following property: above a certain $y=h_{0}$ strictly less than the height of the cell, the cell boundary is the union of smooth, concave curves having curvature bounded away from 0 . Then $P$ is a self-adjoint operator with a positive spectral gap. As a result, there exists a constant $\rho \in(0,1)$ such that for each $\mu \in \mathcal{P}(\mathcal{X})$ with $\|\mu\|_{\pi}<\infty$,

$$
\left\|\mu P^{n}-\pi\right\|_{v} \leq M_{\mu} \rho^{n}
$$




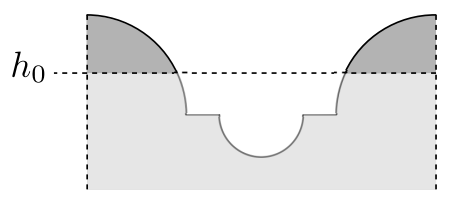

Figure 3: An example of billiard cell for which Theorems 1 and 2 hold. Other than being bilaterally symmetric, its shape is essentially arbitrary below a line $y=h_{0}$ whereas above it, the boundary consists of two smooth concave lines with curvature bounded below by some positive number $K$.

for some constant $M_{\mu}<\infty$ and $n \geq 1$.

Figure 3 gives an example of billiard cell for which Theorem 1 holds.

\subsection{Central Limit and Diffusivity}

Referring back to Figure 1, one expects for a sufficiently long channel that the molecular random flight can be approximated by a Wiener process whose variance corresponds to the Knudsen self-diffusivity. This is justified by a Central Limit Theorem (CLT). This diffusivity has a convenient expression when the transition operator $P$ is self-adjoint. We describe this expression here and prove further details later in the paper.

Let $\left(X_{n}\right)_{n \geq 1}$ be, as above, the stationary Markov chain generated by $P$, with stationary probability measure $\pi$. Recall that $X_{n}$ has values in the space of post-collision velocities $X$. This space can be parametrized by the values of the cosine of the angle the velocity vector makes with the horizontal reference line $y=c$. (See Figure 2.) Thus we may set $\mathcal{X}=(-1,1)$. Let $f: X \rightarrow \mathbb{R}$ be the observable

$$
\tilde{f}(x)=2 r x\left(1-x^{2}\right)^{-1 / 2}
$$

where $r$ is the radius of the channel. We suppose, in the context of formulating a CLT for molecular trajectories, that the length of the channel is infinite. Note that $\tilde{f}\left(X_{n}\right)$ is the distance travelled by the particle along the channel's horizontal axis between the $n$th and the $n+1$ st collisions with the channel wall. The total horizontal displacement up to the $n$th collision is

$$
S_{n}(\tilde{f})=\sum_{k=0}^{n-1} \tilde{f}\left(X_{k}\right)
$$

In its standard form, the CLT gives a limit in distribution for expressions of the form $S_{n}(f) / \sqrt{n}$ where $f$ is an observable having mean zero and finite variance. A simple calculation shows that the horizontal displacement function $\tilde{f}$ has mean zero but infinite variance. For this reason we consider instead the following modified, cut-off displacement observable:

$$
f_{a}(x):=\tilde{f}(x) \mathbb{1}_{\{|\tilde{f}| \leq a\}}(x)+a \mathbb{1}_{\{|\tilde{f}|>a\}}(x)
$$

for large $a>0$. Here $\mathbb{1}_{I}(x)$ denotes the indicator function of the set $I$, which is defined as $\mathbb{1}(x)=1$ if $x \in I$ and 0 if $x \notin I$. There are a number of physical mechanisms that could be invoked to make this cut-off plausible. For example, the channel might have a slight curvature along its length, setting an upper bound on the horizontal distance traveled. See [4] for an 
outline of other mechanisms. We should also note that while the CLT with the usual scaling does not hold for the observable $\tilde{f}$, the distribution of $\tilde{f}\left(X_{n}\right)$ is still in the domain of attraction of the Gaussian law. One can check that $\tilde{f}$ is slowly varying and, as a result, a CLT with nonstandard scaling holds for random billiard Markov chains with sufficient mixing. See [7] for a detailed study of such Markov chains. The program we outline in this paper to estimate the diffusivity should hold in the infinite variance case as well, but we have chosen to focus on the finite variance case for the sake of clarity of exposition. It should also be noted that for cylindrical channels in dimension 3 (and higher), the observable that gives the distance traveled along the axis of the channel is of finite variance.

We suppose the microstructure satisfies the same geometric assumptions of Theorem 1. In particular, $P$ is self-adjoint and has positive spectral gap. Let $\Pi$ be the spectral resolution of $P$ - the projection-valued measure on the spectrum $\sigma(P) \subset[-1,1]$ granted by the Spectral Theorem for bounded self-adjoint operators. Then

$$
P=\int_{-1}^{1} \lambda \Pi(d \lambda)
$$

Let $f$ be any observable in $L_{0}^{2}(X, \pi)$ (for example, the truncated displacement function $f_{a}$ ) and define the measure $\Pi_{f}$ supported on $\sigma(P) \backslash\{1\}$ by

$$
\Pi_{f}(d \lambda):=\langle f, \Pi(d \lambda) f\rangle_{\pi} .
$$

The following is a special case of a theorem that will be stated and proved in Section 4

Theorem 2. Let $\left(X_{n}\right)_{n \geq 0}$ be a Markov chain taking values in $X$ with Markov transition operator $P$ and stationary measure $\pi$. Suppose $P$ is associated to a billiard cell satisfying the same geometric assumptions of Theorem 1 . Let $f \in L_{0}^{2}(X, \pi)$. Then $S_{n}(f) / \sqrt{n}$ converges in distribution to a centered Gaussian random variable $\mathcal{N}\left(0, \sigma_{f}^{2}\right)$, where the variance is given by

$$
\sigma_{f}^{2}=\int_{-1}^{1} \frac{1+\lambda}{1-\lambda} \Pi_{f}(d \lambda)=\langle f, f\rangle_{\pi}+2\left\langle f, P(I-P)^{-1} f\right\rangle_{\pi} .
$$

The expression for the diffusivity given above suggests the following approach for computing $\sigma_{f}^{2}$. Let $L:=P-I$ be the Markov Laplacian and $g$ the solution to the Markov-Poisson equation $L g=-f$. Then the dimensionless Knudsen self-diffusivity coefficient takes the form

$$
\eta=\frac{\sigma_{f}^{2}}{\sigma_{0}^{2}}=1+2\|f\|_{\pi}^{-2}\langle f, P g\rangle_{\pi},
$$

where $\sigma_{0}^{2}=\|f\|_{\pi}^{2}$ is the diffusivity for the process with independent post-collision velocities with the identical distribution $\pi$. In the next subsection we explain one approach to carrying out this program by approximating $L$ by an elliptic differential operator $\mathcal{L}$ whose spectral theory is well understood. It turns out that $\mathcal{L}$ has a canonical form as we show next.

\subsection{The Legendre Equation and Diffusion Approximation}

Our aim now is to show that it is possible to approximate the solution of the Markov-Poisson equation $L g=-f$ for a large class of random billiard microstructures when $P$ is close to the 
identity operator $I$. We consider families of microstructures indexed by a scalar quantity $h$ that, in a sense to be made precise, characterizes a key geometric feature of the microscopic billiard cell, namely its flatness. For each microstructure with parameter $h$, the corresponding Markov operator $P_{h}$ defines the dynamics of the random billiard Markov chain as discussed previously. The key idea now is that for small values $h$, the operator $P_{h}$ will act nearly like the identity operator, due to the flatness of the geometry; the Markov-Laplace operator $L_{h}:=P_{h}-I$, in the limit as $h \rightarrow 0$ and under some general assumptions on the microscopic billiard cell, will then have a canonical approximation by the classical Legendre differential operator, whose spectral theory is well understood. In the rest of the subsection, we make explicit the necessary assumptions on the geometry and give the statement of our operator approximation result and provide examples.

Let the boundary of the billiard cell be the graph of a periodic function $F: \mathbb{T} \rightarrow \mathbb{R}$. (See Figure 2.) In order to characterize how flat the microstructure boundary is, we consider the normal vector field $\mathrm{m}: \mathbb{T} \rightarrow \mathbb{R}^{2}$ along the graph of $F$, and let $\overline{\mathrm{n}}=\overline{\mathrm{n}}(r)$ denote its projection onto its first (horizontal) component. Finally, we let

$$
h:=\int_{\mathbb{T}} \overline{\mathrm{n}}^{2} d r=\int_{\mathbb{T}} \frac{F^{\prime}(r)^{2}}{1+F^{\prime}(r)^{2}} d r .
$$

It will be seen in examples that $h$ captures information about the curvature of the boundary. For small values of $h$, the collision events with the boundary will be relatively simple, often resulting in only a single collision with the cell's boundary and only a small deviation from specular reflection. This implies little change in the tangential momentum of the particle with high probability. It is in this sense that $h$ can be thought to have a role similar to the accommodation coefficient $\vartheta$ referred to earlier in the paper.

Let $X=(-1,1)$ and let $\mathcal{L}$ denote the differential operator acting on smooth functions $f: X \rightarrow \mathbb{R}$ as

$$
\mathcal{L} f(x)=\frac{d}{d x}\left(\left(1-x^{2}\right) \frac{d}{d x} f(x)\right) .
$$

Theorem 3. Let $\left(F_{h}\right)_{h>0}$ be a family of piecewise smooth functions $F_{h}: \mathbb{T} \rightarrow \mathbb{R}$ defining bilaterally symmetric billiard cells, indexed by the flatness parameter $h$ introduced in (3). Let $\left(P_{h}\right)_{h>0}$ be the corresponding Markov transition operators. Then for any $f \in C^{3}(X)$,

$$
L_{h} f(x)=2 h \mathcal{L} f(x)+O\left(h^{3 / 2}\right)
$$

holds for each $v$ such that every initial condition with velocity $v$ results in a trajectory that collides only once with the boundary of the cell.

In the context of Theorem 3 we observe that, for each $x \in \mathcal{X}$, every initial condition with velocity $x$ results in a trajectory that collides only once cell boundary as long as we take $h$ to be sufficiently small.

The differential operator $\mathcal{L}$ has a well understood spectral theory that will be used to obtain information about $P_{h}$. We recall that the eigenvalue problem $\mathcal{L} f=\lambda f$ has square integrable solutions if and only if $\lambda$ is of the form $\lambda=-l(l+1)$ for integers $l \geq 0$. The associated eigenfunctions are the Legendre polynomials $\phi_{l}, l \geq 0$,

$$
\phi_{0}=1, \quad \phi_{1}(x)=x, \quad \phi_{2}(x)=\left(3 x^{2}-1\right) / 2, \ldots
$$


The collection $\left(\phi_{l}\right)_{l \geq 0}$ forms a complete orthogonal basis for $L^{2}(X, \pi)$ and

$$
\left\langle\phi_{n}, \phi_{m}\right\rangle_{\pi}=\int_{x} \phi_{n}(x) \phi_{m}(x) \pi(d x)=\frac{1}{2 n+1} \delta_{n, m},
$$

where $\delta_{n, m}$ is the Kronecker delta symbol.

As a first application of the approximation given in Theorem 3, we give an informal estimation of the spectral gap $\gamma_{h}$ of $P_{h}$ for values of $h$ near 0 . Note that the largest eigenvalue of $P_{h}$ is 1 , with eigenfunctions given by the constant functions. So $\gamma_{h}$ is given by $1-\lambda$ where $\lambda$ is the second largest eigenvalue of $P_{h}$. Using the approximation in Theorem 3 .

$$
P_{h} \phi_{l}=(1-2 h l(l+1)) \phi_{l}+O\left(h^{3 / 2}\right),
$$

where $\phi_{l}$ is the Legendre polynomial associated to eigenvalue $-l(l+1)$. This suggests that the second largest eigenvalue $\lambda$ of $P_{h}$ is given by $\lambda \approx 1-4 h$. Equivalently, this suggests the following asymptotic estimate of $\gamma_{h}$ :

$$
\gamma_{h} \approx 4 h \text {. }
$$

The idea then will be to use the approximation $\mathcal{L}$ of the Markov-Laplacian $L$ in order to give an approximation of the function $g=(I-P)^{-1} f$ that appears in the equation

$$
\sigma_{f}^{2}=\langle f, f\rangle_{\pi}+2\left\langle f, P(I-P)^{-1} f\right\rangle_{\pi}
$$

obtained in Theorem 2. Note that $g$ is a solution of the Markov-Poisson equation $L g=-f$. The following thorem shows that a series solution of the Poisson equation for $\mathcal{L}$ can be given explicitly in terms of Legendre polynomials.

Theorem 4. Let $\left(P_{h}\right)_{h>0}$ be a family of random billiard Markov transition operators for a family of billiard cells satisfying the geometric assumptions of Theorems 2 and 3 . For any function $f \in L_{0}^{2}(X, \pi)$, let $\sigma_{f, h}^{2}$ denote the diffusivity corresponding to $P_{h}$. Then

$$
\sigma_{f, h}^{2}=-\langle f, f\rangle_{\pi}+\frac{1}{h} \sum_{l=1}^{\infty} \frac{2 l+1}{l(l+1)}\left\langle\phi_{l}, f\right\rangle_{\pi}^{2}+O\left(h^{1 / 2}\right) .
$$

Remark 1. It should be noted that for the sake of numerical computations, it is natural to consider the quantity given by truncating the series in (14) after a fixed number of terms $n \geq 1$, so that

$$
\sigma_{f, h}^{2}=-\langle f, f\rangle_{\pi}+\frac{1}{h} \sum_{l=1}^{n} \frac{2 l+1}{l(l+1)}\left\langle\phi_{l}, f\right\rangle_{\pi}^{2}+E_{h, n},
$$

where $E_{h, n}$ is the tail of the series along with the $O\left(h^{1 / 2}\right)$ error term. This quantity can be estimated as follows:

$$
E_{h, n}=\frac{1}{h} \sum_{l=n+1}^{\infty} \frac{2 l+1}{l(l+1)}\left\langle\phi_{l}, f\right\rangle_{\pi}^{2}+O\left(h^{1 / 2}\right) \leq \frac{\|f\|_{\pi}^{2}}{h} \sum_{l=n+1}^{\infty} \frac{2 l+1}{l(l+1)}\left\|\phi_{l}\right\|_{\pi}^{2}+O\left(h^{1 / 2}\right)=\frac{\|f\|_{\pi}^{2}}{h(n+1)}+O\left(h^{1 / 2}\right) .
$$


The theorem implies that the dimensionless self-diffusivity coefficient satsifies

$$
\eta_{f}=-1+\frac{1}{h} \sum_{l=1}^{\infty} \frac{2 l+1}{l(l+1)}\left\langle\phi_{l}, f /\|f\|_{\pi}\right\rangle_{\pi}^{2}+O\left(h^{1 / 2}\right)=-1+\frac{1}{h} C_{f}+O\left(h^{1 / 2}\right),
$$

where $C_{f}$ is defined by this identity. Thus, for small $h$,

$$
\eta_{f} \approx \frac{C_{f}-h}{h} .
$$

Then the approximate identity (5) suggests

$$
\eta_{f} \approx \frac{4 C_{f}-\gamma}{\gamma}
$$

It is interesting to compare this expression with the one obtained under the Maxwell-Smoluchowski model:

$$
\eta=\frac{2-\vartheta}{\vartheta}
$$

where $\vartheta$ is the accommodation coefficient, defined as the fraction of diffuse collisions. We thus obtain a conceptual relation linking the purely geometric quantity $h$ (flatness), the spectral quantity $\gamma$ (spectral gap), and the tangential momentum accommodation coefficient $\vartheta$ defined for a standard and widely used collision model. Finally, it is worth comparing these expressions with the exact equation

$$
\eta_{f}=\int_{-1}^{1} \frac{2-\vartheta}{\vartheta} \bar{\Pi}_{f}(d \vartheta)
$$

where $\bar{\Pi}_{f}(d \vartheta)=\Pi_{f}(d \vartheta) /\|f\|_{\pi}^{2}$, which is obtained from Theorem 2 by setting $\vartheta=1-\lambda$.

\subsection{TwO EXAMPLES}

Consider the microscopic billiard cell, which we will refer to as the small bumps microstructure throughout the discussion, whose boundary is given by arcs of circles as in Figure 4 . The geometric parameter of interest here is the dimensionless curvature given by $K=\ell / R$, where $R$ is the radius of one of the arcs and $\ell$ is the length of the opening to the billiard cell as shown in the figure. An elementary computation using (3) gives

$$
h=\frac{K^{2}}{12} .
$$

As a result, the spectral gap, approximated for values of $K$ near zero, is given by

$$
1-\lambda \approx 4 h=K^{2} / 3 .
$$

Figure 5 shows the numerically obtained values for the spectral gap and $\eta$ compared to the respective approximations as functions of the dimensionless curvature parameter $K$.

A similar computation can be done for the microgeometry in Figure 6 that consists of a mixture of the small bumps geometry together with flat, specularly reflecting lines. In this case, the family is parameterized by the proportion of initial positions $\alpha$ that result in reflections 


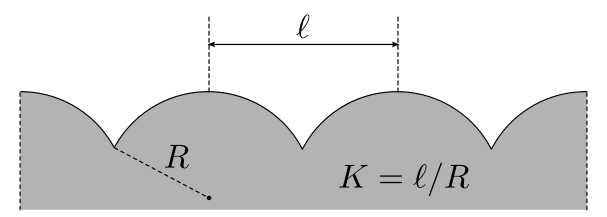

Figure 4: The bumps microstructure with dimensionless curvature parameter $K$.
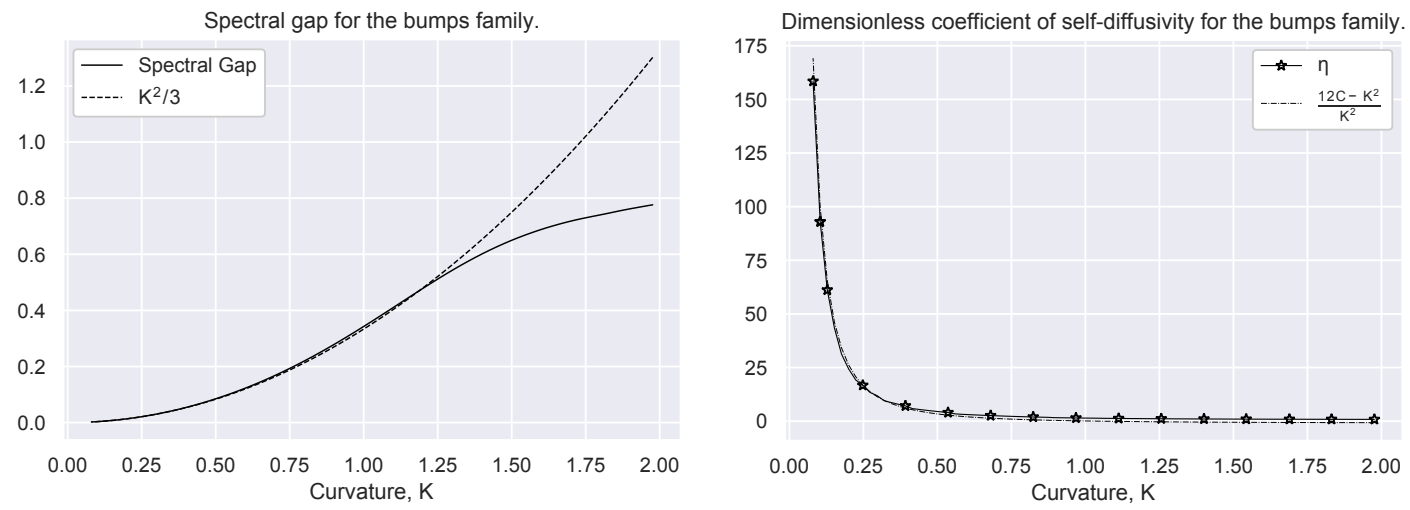

Figure 5: Left: the spectral gap of the operator $P$ for the bumps family of microstructures depicted in Figure 4, with dimensionless curvature parameter $K$, compared with the approximation of the Markov-Laplacian by the Legendre differential operator. The solid curve is constructed from the numerical approximation detailed in Section 2.6. Right: comparison of the dimensionless diffusivity coefficient $\eta$ obtained using (2) and a finite dimensional approximation of $P$ (indicated on the graph by the stars) and the approximation of $\eta$ as a function of the geometric parameter given by (7). The observable is $f_{a}$ with cut-off $a=50000$.

with the part of the boundary with curvature. After expressing the boundary as the graph of an appropriately defined function and computing an elementary integral, we get that $h=\alpha / 3$.

Generalizing this second example, consider the transition operator

$$
P_{\alpha}=\alpha P_{1}+(1-\alpha) I
$$

where $P_{1}$ is the operator associated to a given microstructure. Then $P_{\alpha}$ is associated to the microstructure for which a segment of horizontal line of length $d$ is added to the billiard cell of the first microstructure. The parameter $\alpha$ is then the probability that an incoming particle will not collide with the flat segment. It is easy to see the effect of the additional parameter $\alpha$. Note that $P_{\alpha}-I=\alpha\left(P_{1}-I\right)$. An elementary algebraic manipulation starting from the expression

$$
\sigma_{f, \alpha}^{2}=\langle f, f\rangle_{\pi}+2\left\langle P_{\alpha} f,\left(I-P_{\alpha}\right)^{-1} f\right\rangle_{\pi}
$$

gives

$$
\eta_{f, \alpha}=\eta_{f, 1}+\frac{2(1-\alpha)}{\alpha} \frac{\left\langle f,\left(I-P_{1}\right)^{-1} f\right\rangle_{\pi}}{\|f\|_{\pi}^{2}}
$$


where $f$ is arbitrary. As it is to be expected, $\eta_{f, \alpha}$ approaches infinity as the probability of specular reflection increases to 1 .

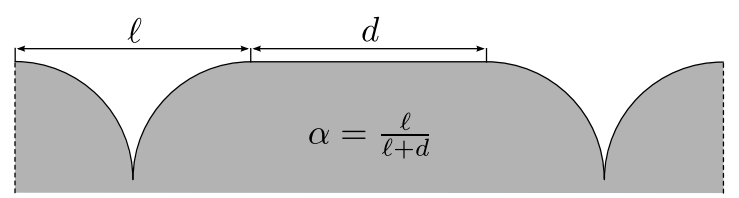

Figure 6: Adding a flat segment to a given microstructure, as indicated in this diagram, gives the transition operator $P_{\alpha}=\alpha P_{1}+(1-\alpha) I$, where $P_{1}$ is the operator associated to the original microstructure.

\subsection{Summary of the numerical TECHniques And EXAmples}

In equation (14) of Theorem 4, we have given our main numerical approach of the paper with respect to analyzing the regime of small flatness parameter $h$; namely, we estimate the dimensionless self-diffusivity $\eta=\eta_{f}$ by truncating the series in equation (14). In this subsection we outline an additional numerical approach for computing the dimensionless self-diffusivity $\eta$ (or, equivalently, the variance $\sigma_{f}^{2}$ of the Gaussian limit of the random flight in a channel). This method, which we will refer to as the Galerkin method, requires us to introduce a finite rank approximation, or discretization, of the Markov operator $P$, which we describe below. The purpose of introducing this additional approach is two-fold. First, the Galerkin method serves as numerical verification of the main approach of using equation (14). Additionally, the Galerkin method is applicable to microstructures which fall outside of the small $h$ regime. As we will see, however, the method has the disadvantage of requiring a discretization of $P$ and, for this reason, is more computationally demanding. We conclude this subsection with a discussion of some additional examples that show the subtle relationship between the spectral gap, the dimensionless self-diffusivity, and geometric features of the microstructure.

The starting point in computing $\eta$ is the equation $\sigma_{f}^{2}=\langle f, f\rangle_{\pi}+2\left\langle f, P(I-P)^{-1} f\right\rangle_{\pi}$, which in turn requires that we obtain the solution $g$ to the Markov-Poisson equation $(P-I) g=-f$. The classical Galerkin method gives us a general approach for solving this equation as follows (see 2] for a broader discussion of the approach). For each $n \geq 1$, let $T_{n}: L_{0}^{2}(X, \pi) \rightarrow R_{n}$ denote the orthogonal projection to the linear span $R_{n}=\left\{\phi_{1}, \ldots, \phi_{n}\right\}$ of Legendre polynomials defined on $X$. Define $g_{n} \in L_{0}^{2}(X, \pi)$ to be the solution of the finite dimensional linear system

$$
\left(I-T_{n} P\right) g_{n}=T_{n} f
$$

Equivalently, we find $g_{n} \in R_{n}$ so that $\left\langle(I-P) g_{n}, \psi\right\rangle_{\pi}=\langle f, \psi\rangle_{\pi}$ for all $\psi \in R_{n}$, which can be done as follows. Writing $g_{n}=\sum_{j=1}^{n} \alpha_{j} \phi_{j}$ and defining $x=\left(\alpha_{1}, \ldots, \alpha_{n}\right)^{\top}, y=\left(\left\langle f, \phi_{1}\right\rangle_{\pi}, \ldots,\left\langle f, \phi_{n}\right\rangle_{\pi}\right)^{\top}$, and $G=\left(\left\langle\phi_{j}, \phi_{i}\right\rangle_{\pi}-\left\langle P \phi_{j}, \phi_{i}\right\rangle_{\pi}\right)_{i, j=1}^{n}$, we are left to do two computations. First, we find the entries of the matrix $G$. Second, we find the solution $x$ to the linear system $G x=y$. This then gives the solution $g_{n}$ to the finite dimensional linear system, and from it the approximate value $\sigma_{\mathrm{GM}, n}^{2}$. The following theorem provides an error estimate for this approximation. A proof is given in Section 5 Figure 7 gives numerical verification of the convergence and error bound for $\sigma_{\mathrm{GM}, n}^{2}$ as given in the theorem. 
Theorem 5. Let $f \in L_{0}^{2}(X, \pi)$, where $X=(-1,1)$, be such that the first derivative $f^{\prime}$ is absolutely continuous and the second derivative $f^{\prime \prime}$ is of bounded variation. Let $\sigma_{f}^{2}$ be defined by the equation

$$
\sigma_{f}^{2}=\langle f, f\rangle_{\pi}+2\left\langle P f,(I-P)^{-1} f\right\rangle_{\pi} .
$$

Then $\lim _{n \rightarrow \infty} \sigma_{\mathrm{GM}, n}^{2}=\sigma_{f}^{2}$. Moreover, we have the following rate of convergence:

$$
\left|\sigma_{f}^{2}-\sigma_{\mathrm{GM}, n}^{2}\right| \leq \frac{C}{4 n-6}
$$

where $C$ is a constant depending on $f$ and $P$ but independent of $n$.

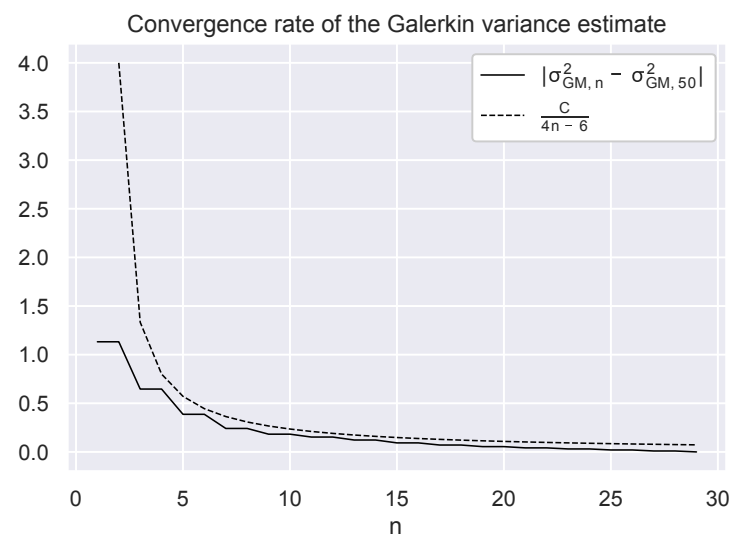

Figure 7: Error bound for the Galerkin approximation of $\sigma_{f}^{2}$ given in Theorem 5

In practice, the drawback in the Galerkin method arises in finding the entries of the matrix $G$. We compute the entries of $G$ by introducing a finite dimensional matrix $P_{M}$ that approximates the Markov operator $P$ and perform numerical integration. We now describe how $P_{M}$ is constructed. Given the billiard cell $\mathcal{N}$ with phase space $\mathcal{V}=\mathbb{T} \times X$, we partition $\mathbb{T}$ and $X$ into $N$ and $M$ evenly spaced subintervals $\left\{I_{1} \ldots, I_{N}\right\}$ and $\left\{J_{1} \ldots, J_{M}\right\}$, respectively. For each subinterval in the partitions, we choose a representative element, e.g. the midpoint, to construct the sequences $\left\{r_{k}\right\}_{k=1}^{N}$ and $\left\{x_{\ell}\right\}_{\ell=1}^{M}$, respectively. For each pair $(r, x)$ in the set $\left\{\left(r_{k}, x_{\ell}\right): 1 \leq k \leq N, 1 \leq \ell \leq M\right\}$ we then simulate the standard billiard motion of a particle in the cell $\mathcal{M}$ with initial conditions $(r, x)$ and record the particle's velocity upon its return to $\mathbb{T}$. The finite rank approximation $P_{M}$ is then the $M \times M$ matrix whose $i j$ entry is the proportion of $N$ trajectories whose initial velocity $x_{i} \in J_{i}$ yield a return velocity in the subinterval $J_{j}$.

We mention here that $\sigma_{G M, n}^{2}$ stabilizes for moderately sized values of $M$ (say $M=1000$ ), independent of the choice of $n$. Moreover, we have used the matrix $P_{M}$ to give another numerical approximation of $\sigma_{f}^{2}$. For larger values of $h$, it is possible to solve the Markov-Poisson equation $\left(P_{M}-I\right) g=-f$ using a standard numerical linear system solver (which implements the biconjugate stabilized method, or BiC method). We should mention that the BiC method is used simply to give numerical verification for the use of the Galerkin method in the large $h$ regime. For small values of $h$, the spectral gap of $P_{M}$ is small and the condition number of $I-P_{M}$, 
propositional to the inverse of the spectral gap of $P_{M}$, is too high to be reliable. In Figure 8 , we have shown a comparison of approximations for $\sigma_{f}^{2}$ for the small bumps family introduced in the previous subsection and the observable $f_{a}$ defined in (1) as produced by the three methods: (1) using equation 14 truncated to $n$ terms and denoted by $\sigma_{\mathrm{Lser}, n}^{2},(2)$ using the Galerkin method with dimension $n$ and denoted by $\sigma_{\mathrm{GM}, n}^{2}$, and (3) using the BiC linear system solver.
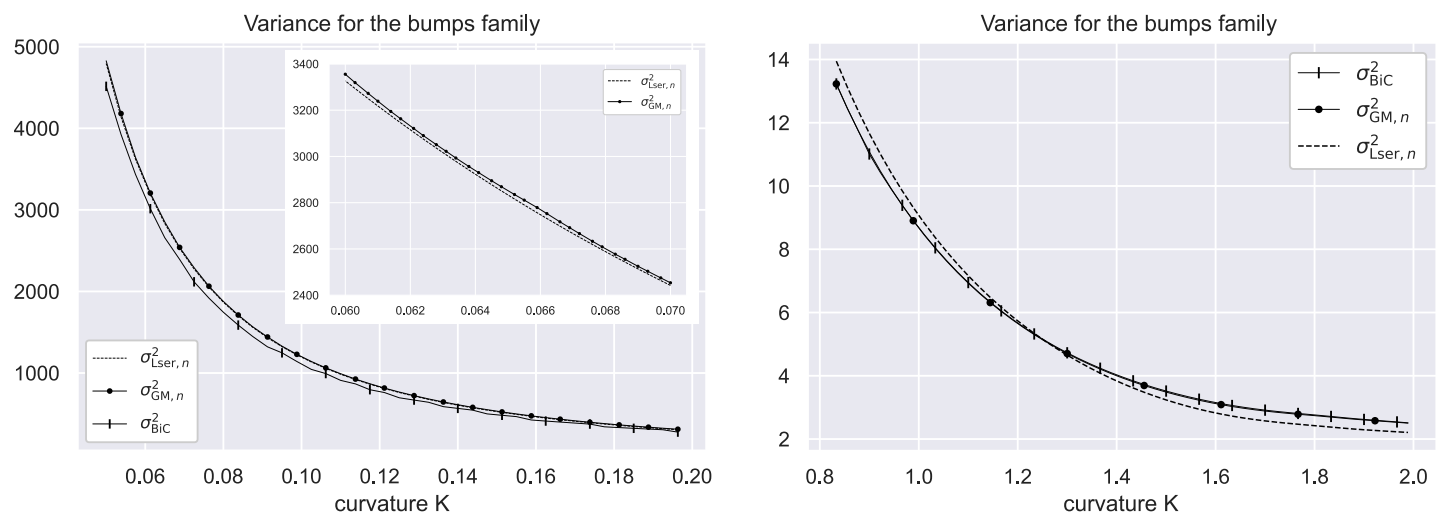

Figure 8: Comparison of the variance, computed using the methods described in this section, for the simple bumps family with observable $f$ given by the horizontal displacement function along the length of the channel with cut-off $a=50000$ (there are no qualitative differences for larger choices of the cut-off). The value of $\sigma_{\mathrm{Lser}, n}^{2}$ is computed using (14) where we have used the first $n=500$ terms in the series. For $\sigma_{\mathrm{GM}, n}^{2}$ we have used dimension $n=200$ and a finite rank approximation $P_{M}$ of $P$ with $M=1000$. The same matrix $P_{M}$ has been used for $\sigma_{\mathrm{BiC}}^{2}$. On the left, the dimensionless curvature parameter $K$ is relatively small, while on the right it is relatively large. The inset of the graph on the left zooms in on the smallest values of $K$, where the $\sigma_{\mathrm{Lser}, n}^{2}$ and $\sigma_{\mathrm{GM}, n}^{2}$ approximations are valid.

We conclude the section with the result of two more numerical experiments. A first example is given by the family of microstructures depicted in Figure 9 There are two competing curvatures, which are fixed while the height parameter $d$ varies over a range of positive and negative values. When $d<0$, the higher curvature bump is more exposed and when $d>0$ the smaller curvature bump is on top.

The numerical results are shown in the plots of Figure 10. The interpretation is somewhat straightforward: when the bigger curvature bump is more exposed to collision with the particles, scattering is more diffuse, spectral gap is larger, and diffusivity is smaller (slower diffusion), than when the less curved bump rises above the other. Perhaps more surprising is the near perfect mirror symmetry between the two graphs.

In the second example we obtain the dimensionless diffusivity and spectral gap for the oneparameter family of microstructures indicated in Figure 11. Here the parameter investigated is the (dimensionless) width of the flat top wall, while the radius $R$ of the curved part is kept constant. Diffusivity is computed using the Galerkin method (dimension 200) while the spectral gap is obtained more directly by computing eigenvalues of the finite dimensional approximation of $P$.

The results are now somewhat harder to interpret. The interplay between the flat wall top, the curvature of the middle bumps, and reflection on the sides of the walls creates a qualitatively 


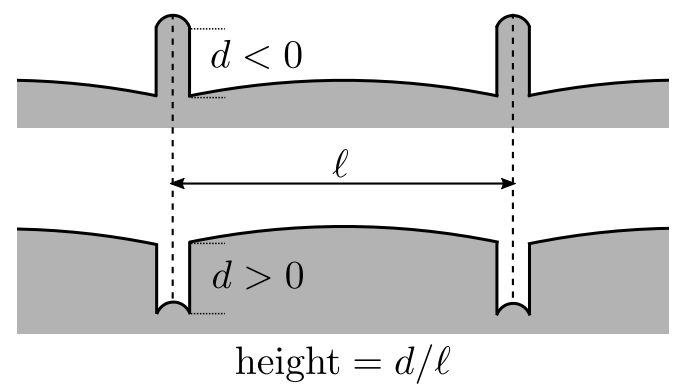

Figure 9: The two-bumps family. By varying the parameter $d$ keeping the curvatures constant, we can investigate how the two curvatures compete against each other in the determination of the spectral gap and the dimensionless coefficient of self-diffusivity $\eta$. The result is shown in Figure 10

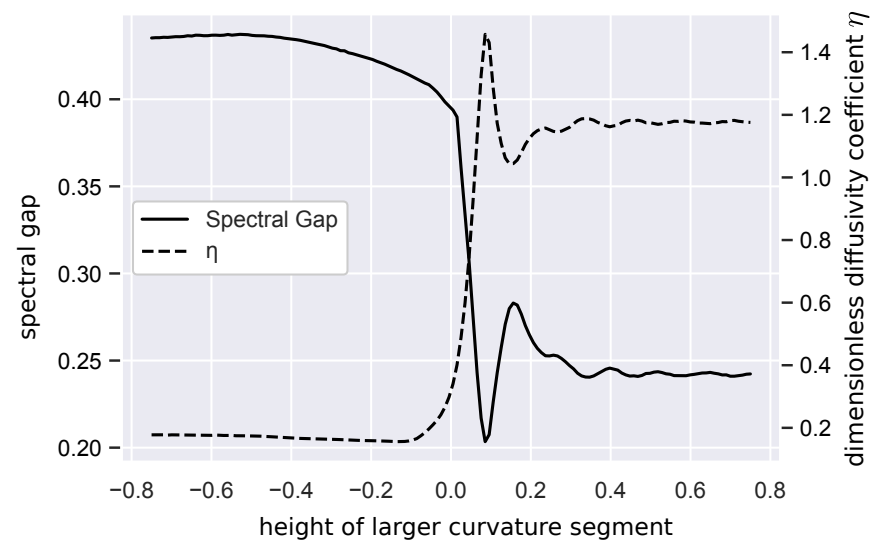

Figure 10: Spectral gap and dimensionless coefficient of self-diffusivity for the microstructure of Figure 9

more complicated effect. Nevertheless, both this and the previous example show a marked transition in the values of diffusivity and spectral gap as the height of the wall (with curved top in the first example and flat top in the second) crosses the height of the adjacent curved segments. Once again, we observe near mirror symmetry in the graphs of spectral gap and diffusivity as functions of the geometric parameter. This is an interesting observation that merits further investigation.

\section{Spectral Gap AND ERGODICITy}

The theorems of the previous sections will be strengthened and proved in this and following sections. We begin this section by introducing a useful technique for decomposing the operator $P$. The idea will be to condition on the event that a billiard trajectory within the microscopic cell satisfies certain properties, which will allow us to focus attention on geometric features of the microgeometry that create mixing in the dynamics. More specifically, we show here that 


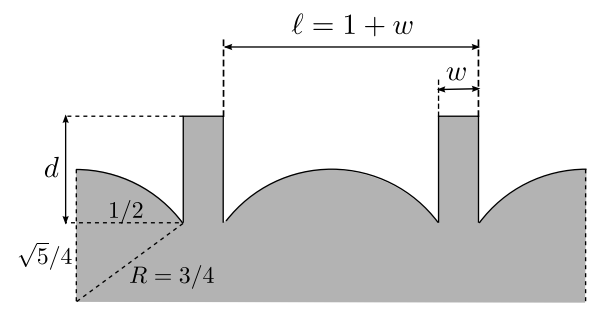

Figure 11: Bumps with flat top wall microstructure. The geometric parameters we vary are the relative width $w$ and height $d$ of the flat top wall.

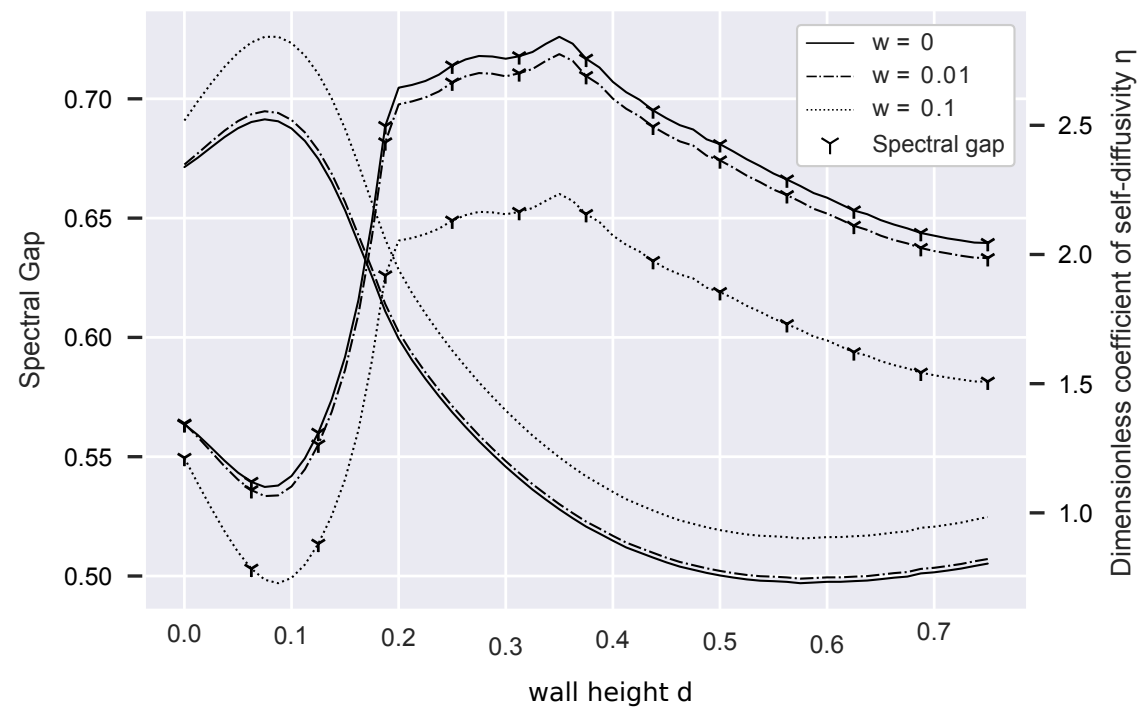

Figure 12: Spectral gap and diffusivity coefficient $\eta$ for the microstructure shown in Figure 11. The geometric parameters being varied are the relative height $d$ of the flat top wall and its relative width $w$.

under assumptions to be stated, the transition probability operator $P$ for the random billiard Markov chain has a spectral gap by showing that for certain components of the decomposition it is a Hilbert-Schmidt operator. This, along with an additional geometric assumption that yields a reversible Markov chain, in turn will give ergodicity.

Let $N:=\mathbb{T} \times X$ be the space of initial conditions of a scattering event and let $N_{1}, N_{2}, \ldots$ be a measurable partition of $N$. For each $x \in \mathcal{X}$ and $i \geq 1$, let $N_{i}(x):=\left\{r \in \mathbb{T}:(r, x) \in N_{i}\right\}$. Define $\alpha_{i}(x):=\left|N_{i}(x)\right|$, where $|\cdot|$ denotes the size of a set under the normalized Lebesgue measure on $\mathbb{T}$. For each $f \in L^{2}(X, \pi)$, define

$$
\left(P_{i} f\right)(x)= \begin{cases}\frac{1}{\alpha_{i}(x)} \int_{N_{i}(x)} f(X(r, x)) d r & \text { if } \alpha_{i}(x) \neq 0 \\ 0 & \text { if } \alpha_{i}(x)=0 .\end{cases}
$$

We refer to $P_{i}$ as the conditional operator associated to partition element $N_{i}$. Note that $P_{i} \mathbb{1}_{A}(x)$ 
is the conditional probability that the outgoing velocity vector is in $A \subset \mathcal{X}$ given pre-collision velocity $x$ and given that the event $N_{i}$ holds. Let $\pi_{i}$ denote the measure on $X$ such that $\pi_{i}(d x)=\alpha_{i}(x) /(d r \otimes \pi)\left(N_{i}\right) d x$. Then $\pi_{i}$ is the conditional measure given by $\pi$ conditioned on the event that $N_{i}$ holds. Finally, observe that for any $f \in L^{2}(X, \pi)$, it makes sense to decompose $P$ as follows:

$$
(P f)(x)=\sum_{i} \alpha_{i}\left(P_{i} f\right)(x) .
$$

We now outline some properties of the conditional operators and the resulting decomposition of $P$. For details of proofs, see 14 .

Proposition 2. Let $P_{i}, i \geq 1$, be the conditional operators associated to the measurable partition $N_{1}, N_{2}, \ldots$ of the space $N$ of initial conditions of billiard trajectories within billiard microcell $M$, and let $\pi_{j}$ be the conditional measures associated to the partition. Then for each $i \geq 1$,

1. $P_{i}$ has norm 1.

2. Each term $\alpha_{i} P_{i}$ in the decomposition has norm at most $\left\|\alpha_{i}\right\|_{\infty}$.

3. If $N_{i}$ is symmetric - that is, it is invariant under the map $(r, x) \mapsto(1-r, J x)$ where Jx denotes the reflection across the vertical axis in $\mathbb{H}_{-}^{2}$ of the velocity vector corresponding to $x$ and $\mathbb{T}$ is identified with the unit interval - then $P_{i}$ is self-adjoint as an operator on $L^{2}\left(X, \pi_{i}\right)$.

The following assumptions will be shown to be sufficient for ergodicity.

Assumption 1. The billiard cell is symmetric with respect to reflection across the vertical axis given by the map $(x, y) \mapsto(-x, y)$.

Assumption 2. There exists a measurable partition $N_{1}, N_{2}, \ldots$ whose elements are symmetric and such that the following holds for at least one partition element $N_{j}$.

1. The trajectories with initial conditions in $N_{j}$ collide only with portions of the boundary of the microscopic billiard cell consisting piecewise smooth concave curves whose curvatures are bounded below by a constant $K>0$.

2. $\inf _{v \in X} \alpha_{j}(v)>0$.

Note that these assumptions are not optimal - for example, billiard cells with convex sides have been shown to give geometrically ergodic random billiard Markov chains in [7 — but capture a large class of examples like those in Section 2. The key idea of Assumption 2 is that partitioning the phase space and subsequently decomposing the Markov transition operator into corresponding conditional operators allows us to focus our study of the operator only on the features that create enough dispersion to yield ergodicity.

Theorem 6. Let $P$ be the Markov transition operator for a random billiard Markov chain whose billiard cell satisfies Assumptions 1 and 2 . Then $P$ is a self-adjoint operator with spectral gap. As a result, there exists a constant $\rho \in(0,1)$ such that for each probability measure $\mu \in \mathcal{P}(X)$, absolutely continuous with respect to $\pi$ with $\|\mu\|_{\pi}<\infty$, there exists a constant $M_{\mu}<\infty$ such that $\left\|\mu P^{n}-\pi\right\|_{v} \leq M_{\mu} \rho^{n}$. 
Note that Theorem 6 generalizes Theorem 1. Indeed, for billiard cells that satisfy the geometric property in the hypotheses of Theorem 1, it is clear that for each $x$, there exists an open set $W_{x}^{1} \subset \mathbb{T}$ such that for each $r \in W_{x}^{1}$, the billiard trajectory with initial condition $(r, x)$ results in one collision with the boundary of the billiard cell before returning to the reference line. Letting $N_{1}=\left\{(r, x): x \in \mathcal{X}, r \in W_{x}^{1}\right\}$ and $N_{2}=N \backslash N_{1}$, it is clear that Assumptions 1 and 2 are satisfied. We also note that Theorem 6 includes as a special case, the case of i.i.d. mixtures of microstructures.

The proof of Theorem 6 requires a series of lemmas, which we now introduce. Note that these lemmas are adapted from a series of lemmas in 11 but the present statements have more relaxed hypotheses on the geometry of the billiard cell and thus are stronger.

Before stating the first lemma, we need to introduce some notation. Consider a measurable partition satisfying the conditions in Assumption 2, where $N_{j}$ and $P_{j}$ are the partition element and corresponding conditional operator that satisfy the restrictions in the assumption. Let $W_{x}^{i}:=\left\{r \in \mathbb{T}:(r, x) \in N_{i}\right\}$ for each partition element $N_{i}$. For each $x \in \mathcal{X}$, we let $X_{x}: \mathbb{T} \rightarrow \mathcal{X}$ be the function given by $X_{x}(r)=X(r, x)$, where $X(r, x)$ is the return velocity at the reference line of the billiard cell for a trajectory with initial condition $(r, x)$.

Lemma 1. Suppose the billiard cell satisfies Assumption 2, with partition element $N_{j}$ and conditional operator $P_{j}$ satisfying the conditions in the assumption. Then for all $x \in X$, the set $W_{x}^{j}=\left\{r \in \mathbb{T}:(r, x) \in N_{j}\right\}$ consists of a countable union of open intervals $W_{x, i} \subset \mathbb{T}$. Moreover, the restriction $X_{x, i}:=\left.X_{x}\right|_{W_{x, i}}$ is a diffeomorphism from $W_{x, i}$ onto its image $V_{x, i}$. Finally, when we use the convention $X=(0, \pi)$, we have that for all $f \in L^{2}\left(X, \pi_{j}\right), P_{j} f(x)=\int_{X} f(\phi) \omega(x, \phi) \pi_{j}(d \phi)$ where

$$
\omega(x, \phi):=\frac{(\lambda \otimes \pi)\left(N_{j}\right)}{\alpha_{j}(x) \alpha_{j}(\phi)} \sum_{i} \mathbb{1}_{V_{x, i}}(\phi)\left(\frac{1}{2}\left|X_{x, i}^{\prime}\left(X_{x, i}^{-1}(\phi)\right)\right| \sin \phi\right)^{-1}
$$

and $\mathbb{1}_{V_{x, i}}$ denotes the indicator function of the set $V_{x, i}$.

Proof. We begin by outlining some standard facts in the theory of classical billiards. See [6] for details. Let $\Gamma$ denote the boundary of the billiard cell $Q$ and note that $\Gamma=\bigcup_{i} \Gamma_{i}$ consists of a union of smooth component curves, or walls. We denote by $\Gamma_{0}$ the reference line, which is identified with $\mathbb{T}$. Let $\mathcal{M}=\bigcup_{i} \mathcal{M}_{i}$ be the collision space, where each set $\mathcal{M}_{i}$ consists of pairs $(q, v)$ where $q \in \Gamma_{i}$ and $v$ points into the interior of $Q$. The billiard map $\mathcal{F}: \mathcal{M} \rightarrow \mathcal{M}$ is the map defined so that $\mathcal{F}(q, v)$ gives the pair $\left(q^{\prime}, v^{\prime}\right)$ where $q^{\prime}$ is the first intersection of the ray $q+t v$, $t>0$, with $\partial Q$. The normalized measure $m \otimes \pi \in \mathcal{P}(\mathcal{M})$, where $m$ is the normalized arclength measure on $\partial Q$, is left invariant by $\mathcal{F}$. Moreover, if we let $T: N \rightarrow N$ be the first return map of billiard orbits, the measure $d r \otimes \pi$, where $d r$ is the normalized Lebesgue measure on $\mathbb{T}$, is left invariant by $T$. By Poincaré recurrence, there is a subset $E_{0} \subset N$ of full $d r \otimes \pi$ measure of orbits that start at and return to $\Gamma_{0}$ in a finite number of steps, and the orbits are non-singular, ie. they do not hit corners of boundary and there are no grazing tangential collisions. As a result, for each $(q, v) \in E_{0}$, there is an open neighborhood in $N$ whose elements return to $N$ in the same number of steps as $(q, v)$ and the return map on this set is smooth. In a similar fashion, it follows that the map $X_{x}: \mathbb{T} \rightarrow X$ is smooth on an open subset of $\mathbb{T}$ and its restriction to the set $W_{x}^{j}$ is likewise a diffeomorphism on an open set which consists of a countable union of open intervals $W_{x, i} \subset \mathbb{T}$. It is also the case that for dispersing billiards, e.g. those billiards for which $\partial Q$ consists of smooth convex curves with positive curvature, the restriction $X_{x, i}$ of $X_{x}$ 
to the set $W_{x, i}$ has the property that $X_{x, i}^{\prime} \neq 0$. Moreover, the summation in $\sqrt{10}$ is well defined; see [6, Lemma 5.56].

We conclude the proof with a verification that the function $\omega$ defined in 10 is a kernel for $P_{j}$. Let $A \subset \mathcal{X}$ be a measurable set and let $A_{x, i}=\left\{r \in W_{x, i}: X_{x, i}(r) \in A\right\}$. Then

$$
\begin{aligned}
\int_{A} \omega(x, \phi) \pi_{j}(d \phi) & =\frac{1}{\alpha_{j}(x)} \sum_{i} \int_{A \cap V_{x, i}}\left(\frac{1}{2}\left|X_{x, i}^{\prime}\left(X_{x, i}^{-1}(\phi)\right)\right| \sin \phi\right)^{-1} \pi(d \phi) \\
& =\frac{1}{\alpha_{j}(x)} \sum_{i} \int_{X_{x, i}\left(A_{x, i}\right)}\left(\left|X_{x, i}^{\prime}\left(X_{x, i}^{-1}(\phi)\right)\right|\right)^{-1} d \phi \\
& =\frac{1}{\alpha_{j}(x)} \sum_{i} \int_{A_{x, i}} d r \\
& =P_{j} \mathbb{1}_{A}(x) .
\end{aligned}
$$

Since this relation holds for indicator functions, it follows by a standard argument using linearity and the density of simple functions in $L^{2}\left(X, \pi_{j}\right)$ that $P_{j}$ has kernel $\omega$ for all $f \in L^{2}\left(X, \pi_{j}\right)$.

The next intermediary lemma gives an estimate on the kernel in Lemma 1. Its proof follows from [11, Lemmas 6.5, 6.6, 6.7] with only minor modifications.

Lemma 2. Consider a billiard cell satisfying Assumption 2 and let $\omega$ be the kernel given in 10). Then $\omega \in L^{2}\left(X \times X, \pi_{j} \otimes \pi_{j}\right)$.

The following lemma is adapted from [22, Theorem 9.9]. It will be used to show that for an operator $P$ which admits a decomposition as in (9), it suffices to show that one conditional operator is compact in order to prove that $P$ has spectral gap. The notation $\|\cdot\|$ is used to denote the canonical Hilbert space operator norm.

Lemma 3. Let $K$ and $T$ be bounded self-adjoint operators on a Hilbert space and suppose that $K$ is compact. Then the essential spectrum of $T+K$ is contained in the essential spectrum of $T$. In particular, if $\|T+K\|=1$ and $\|T\|<1$, then the spectral gap of $T+K$ satisfies $\gamma(T+K) \geq \min \{1-\|T\|, \gamma(K)\}$.

We conclude with the proof of the section's main theorem.

Proof of Theorem 6 . That $P$ is self adjoint follows from Assumption 1 and Proposition 1. To see that $P$ has spectral gap, we apply Lemma 3 . Using the notation of the lemma, we let $K=\alpha_{j} P_{j}$ and $T=\sum_{i \neq j} \alpha_{i} P_{i}$. Then, applying Lemmas 1 and 2 , we have that $K$ is a Hilbert-Schmidt integral operator and hence it is compact. It is clear that $T$ is bounded and self-adjoint and $\|T+K\|=\|P\|=1$, where $\|\cdot\|$ is the $L^{2}$-operator norm. Moreover, $1-\|T\| \geq \inf _{v \in X} \alpha_{j}(v)>0$. It follows that the spectral gap of $P$ is strictly positive. The concluding statement of exponential convergence to the stationary measure in total variation then follows immediately using the Cauchy-Schwarz inequality since $\|\mu\|_{v} \leq 1 / 2\|\mu\|_{\pi}$.

\section{Diffusivity}

Let $f: X \rightarrow \mathbb{R}$ be a function on the state space of the random billiard Markov chain $\left(X_{n}\right)_{n \geq 0}$ with Markov transition operator $P$. We refer to $f$ as an observable (or functional) of the 
Markov chain. Without loss of generality, we suppose that it has mean zero with respect to the stationary distribution: $\pi(f)=0$. Our focus in this section will be on the limiting distribution (after appropriate scaling) of partial sums of the functional of the Markov chain given by

$$
S_{n}(f):=\sum_{k=0}^{n-1} f\left(X_{k}\right)
$$

It is well known that under appropriate mixing conditions for the Markov chain, $S_{n}(f) / \sqrt{n}$ converges in distribution to a centered Gaussian distribution with variance parameter $\sigma_{f}^{2}$. As a preliminary result, we show that random billiard Markov chains with microstructure have sufficiently fast mixing for a (central) limit theorem of this kind to hold. However, our primary focus will be to show that the variance $\sigma_{f}^{2}$ of the limiting Gaussian distribution, which we refer to as the diffusivity of the system, can be rigorously approximated, and formulas can be derived in terms of geometric parameters for families of random billiard microstructures.

We use here a result adapted from [16, which states that the central limit theorem holds for reversible Markov chains satisfying a nondegeneracy condition on $\sigma_{f}^{2}$.

Theorem 7. Let $\left(X_{n}\right)_{n \geq 0}$ be a Markov chain with stationary measure $\pi$ and let $f \in L_{0}^{2}(X, \pi)$. If the Markov chain is reversible, then $S_{n}(f) / \sqrt{n}$ converges in distrubtion to a centered Gaussian random variable $\mathcal{N}\left(0, \sigma_{f}^{2}\right)$ as long as $\sigma_{f}^{2}<\infty$, where $\sigma_{f}^{2}$ is given by (11).

In the discussion that follows, it will be useful to express $\sigma_{f}^{2}$ in terms of the spectrum of $P$, viewed as an operator on $L^{2}(X, \pi)$. We first note that since $P$ is a bounded, self-adjoint operator on $L^{2}(X, \pi)$ with norm 1 , there exists a projection-valued measure $\Pi$, supported on the spectrum $\sigma(P) \subset[-1,1]$ of $P$, defined so that

$$
P=\int_{-1}^{1} \lambda \Pi(d \lambda) .
$$

For each $f \in L_{0}^{2}(X, \pi)$, we further define a measure $\Pi_{f}$ supported on $\sigma(P) \backslash\{1\}$ by $\Pi_{f}(d \lambda):=$ $\langle f, \Pi(d \lambda) f\rangle_{\pi}$. Now, observe that

$$
\begin{aligned}
\sigma_{f}^{2} & =\langle f, f\rangle_{\pi}+2 \sum_{k=1}^{\infty}\left\langle f, P^{k} f\right\rangle_{\pi} \\
& =\langle f, f\rangle_{\pi}+2\left\langle f, P(I-P)^{-1} f\right\rangle_{\pi} \\
& =\int_{-1}^{1} \frac{1+\lambda}{1-\lambda} \Pi_{f}(d \lambda) .
\end{aligned}
$$

Using the expression in (13), we show that the existence of a positive spectral gap is sufficient for the central limit theorem to hold.

Corollary 1. Let $\left(X_{n}\right)_{n \geq 0}$ be a Markov chain with Markov transition operator $P$ and stationary measure $\pi$. Let $f \in L_{0}^{2}(X, \pi)$. If the Markov chain is reversible and $P$ has spectral gap $\gamma>0$, then $S_{n}(f) / \sqrt{n}$ converges in distrubtion to a centered Gaussian random variable $\mathcal{N}\left(0, \sigma_{f}^{2}\right)$.

Proof. Since $P$ has spectral gap, there exists $0<\rho<1$ such that for every $\lambda \epsilon \operatorname{supp}\left(\Pi_{f}\right), \lambda \leq \rho$. Therefore, $\sigma_{f}^{2}$, as given by 13, , is finite since $\sigma_{f}^{2} \leq(1+\rho) /(1-\rho) \pi\left(f^{2}\right)<\infty$. 


\subsection{DifFUSION APPROXIMATION AND DIFFUSIVITY}

We now prove Theorem 3 Recall that the boundary of the billiard cell is assumed to be the graph of a periodic function $F: \mathbb{T} \rightarrow \mathbb{R}$. Also recall the definitions of $h$ and $\mathcal{L}$ from Subsection 2.4 .

Proof of Theorem 3. When only a single boundary surface collision occurs, the relationship between the initial and return velocity vectors, $v=\left(x, v_{0}\right)$ and $V(r, v)$ respectively, is straightforward. Indeed, let $\mathrm{m}: \mathbb{T} \rightarrow \mathbb{R}^{2}$ denote the vector field of normal vectors along the boundary of the billiard cell and let $\overline{\mathrm{n}}$ and $\mathrm{m}_{0}$ denote the first (horizontal) and second (vertical) components of $\mathrm{m}$. If collision with the boundary surface occurs at the point $\left(r^{\prime}, F\left(r^{\prime}\right)\right)$, then $V(r, v)=v-2\left\langle v, \mathrm{~m}\left(r^{\prime}\right)\right\rangle \mathrm{m}\left(r^{\prime}\right)$, where $\langle\cdot, \cdot\rangle$ denotes the Euclidean inner product. Note that by elementary geometry

$$
\mathrm{m}\left(r^{\prime}\right)=\frac{1}{\sqrt{1+F^{\prime}\left(r^{\prime}\right)^{2}}}\left(-F^{\prime}\left(r^{\prime}\right), 1\right)^{\top}, \quad r=r^{\prime}-\left(F\left(r^{\prime}\right)-c\right) x / v_{0}
$$

It now follows that for any smooth function $f:(-1,1) \rightarrow \mathbb{R}$

$$
\begin{aligned}
\operatorname{Pf}(x) & =\int_{\mathbb{T}} f\left(x-2\left\langle v, \mathrm{~m}\left(r^{\prime}\right)\right\rangle \overline{\mathrm{n}}\left(r^{\prime}\right)\right) d r \\
& =\int_{\mathbb{T}} f\left(x-2(\alpha+\beta) \overline{\mathrm{n}}\left(r^{\prime}\right)\right)(1+\alpha / \beta) d r^{\prime},
\end{aligned}
$$

where $\alpha=\overline{\mathrm{n}} x, \beta=\mathrm{m}_{0} v_{0}$. Moreover, by Assumption 1, the symmetry relations $\overline{\mathrm{n}}(\ell-r)=-\overline{\mathrm{n}}(r)$ and $\mathrm{m}_{0}(\ell-r)=\mathrm{m}_{0}(r)$ hold. Using these relations, and suppressing the explicit dependence of $\overline{\mathrm{n}}$ on $r^{\prime}$ for the sake of simplicity of notation, and we get

$$
P f(x)=\frac{1}{2} \int_{\mathbb{T}}[f(x-2(\alpha+\beta) \overline{\mathrm{n}})(1+\alpha / \beta)+f(x+2(-\alpha+\beta) \overline{\mathrm{n}})(1-\alpha / \beta)] d r^{\prime} .
$$

From here we use the second order Taylor approximation of $\phi$ centered about $x$. Observe that for $w \in(-1,1)$

$$
f(x+w)=f(x)+f^{\prime}(x) w+\frac{f^{\prime \prime}(x)}{2} w^{2}+R_{x}(w),
$$

where $R$ is the usual Taylor remain term $R_{x}(w)=f^{\prime \prime \prime}(c) w^{3} / 3$ ! for some $c$ in the interval between $x$ and $w$. Using this, together with straightforward algebraic manipulation that we omit for the sake of clarity of exposition, we get that

$$
\begin{aligned}
P f(x) & =f(x)-4 x f^{\prime}(x) \int_{\mathbb{T}} \overline{\bar{n}}^{2} d r^{\prime}+f^{\prime \prime}(x) \int_{\mathbb{T}} \overline{\mathrm{n}}^{2}\left(6 \alpha^{2}+2 \beta^{2}\right) d r^{\prime}+E(x) \\
& =f(x)-4 x f^{\prime}(x) h+2\left(1-x^{2}\right) f^{\prime \prime}(x) h+O\left(h^{2}\right)+E(x) \\
& =f(x)+2 h \frac{d}{d x}\left(\left(1-x^{2}\right) f^{\prime}(x)\right)+O\left(h^{2}\right)+E(x),
\end{aligned}
$$

where $h=\int_{\mathbb{T}} \overline{\mathrm{m}}^{2} d r^{\prime}$ and $E$ is an error term. The error term arises from the remainder $R$ and is bounded as follows: $|E| \leq C_{\phi} p\left(x, v_{0}\right) I_{3}$, where $C_{\phi}$ is a constant that depends only on the third derivative of $\phi, p\left(x, v_{0}\right)$ is a polynomial in $x, v_{0}$ of degree at most 3 with coefficients that do not depend on $\phi$, and $I_{3}:=\int_{\mathbb{T}} \overline{\mathrm{m}}^{3} d r^{\prime}$. 


\subsection{Computing the Diffusivity}

The differential operator $\mathcal{L}$ defined in $(4)$ has a well understood spectral theory. We will take advantage of this in the following to give a method for computing $\sigma_{f}^{2}$. Before going on, we first note a few well known facts about $\mathcal{L}$.

Proposition 3. Let $\mathcal{L}$ be the Legendre differential operator defined in (4). The following properties hold.

1. The eigenvalue problem $\mathcal{L} f=\lambda f$ has solutions if and only if $\lambda$ is of the form $\lambda=-l(l+1)$ for integers $l \geq 0$.

2. The solutions of the eigenvalue problem are the polynomials $\phi_{l}, l \geq 0$, known as the Legendre polynomials. The first few are given by $\phi_{0}=1, \phi_{1}(x)=x, \phi_{2}(x)=\left(3 x^{2}-1\right) / 2$.

3. The collection $\left(\phi_{l}\right)_{l \geq 0}$ of Legendre polynomials form a complete orthogonal basis for $L^{2}(X, \pi)$ and

$$
\left\langle\phi_{n}, \phi_{m}\right\rangle:=\int_{x} \phi_{n}(x) \phi_{m}(x) \pi(d x)=\frac{1}{2 n+1} \delta_{n, m},
$$

where $\delta_{n, m}$ is the Kronecker delta symbol.

We are now ready to discuss the diffusivity $\sigma_{f}^{2}$ introduced at the start of the section. The idea will be to use the diffusion approximation $\mathcal{L}$ of the Markov-Laplacian $L$ in order to give an approximation of the function $g=(I-P)^{-1} f$ that arises in (12). Note that $g$ is a solution of the Markov-Poisson equation $L g=-f$. We first show that a series solution of the classical Poisson equation can be given explicitly in terms of Legendre polynomials.

Lemma 4. For any $f \in L_{0}^{2}(X, \pi)$, the equation $\mathcal{L} g=-f$ has solution given by

$$
g=\sum_{l=1}^{\infty} a_{l} \phi_{l}, \quad a_{l}=\frac{2 l+1}{2 l(l+1)}\left\langle\phi_{l}, f\right\rangle_{\pi} .
$$

Proof. Let $f \in L_{0}^{2}(X, \pi)$. Since the Legendre functions form a complete orthogonal basis for $L_{0}^{2}(X, \pi), f=\sum_{l=1}^{\infty} b_{l} \phi_{l}$, where $b_{l}=(2 l+1)\left\langle\phi_{l}, f\right\rangle_{\pi}$. Now, let $g=\sum_{l=1}^{\infty} a_{l} \phi_{l}$, where $a_{l}=b_{l} /(l(l+1))$. Observe that $\mathcal{L} g=\sum_{l=1}^{\infty} a_{l} \mathcal{L} \phi_{l}=-\sum_{l=1}^{\infty} a_{l} l(l+1) \phi_{l}=-f$.

With the lemma in hand, we now give our main approximation result. The idea of the proof will be to contruct a series solution approximation of the Markov-Poisson equation using the series solution of the Poisson equation along with the diffusion approximation of $P$. We use the estimates in Theorem 3 to control the error terms in our approximation.

Theorem 8. Let $\left(P_{h}\right)_{h>0}$ be a family of random billiard Markov transition operators for a family of microscopic billiard cells satisfying Assumptions 1 and 2. For any function $f \in L_{0}^{2}(X, \pi)$, let $\sigma_{f, h}^{2}$ denote the diffusivity corresponding to $P_{h}$. Then

$$
\sigma_{f, h}^{2}=-\langle f, f\rangle_{\pi}+\frac{1}{h} \sum_{l=1}^{\infty} \frac{2 l+1}{l(l+1)}\left\langle\phi_{l}, f\right\rangle_{\pi}^{2}+O\left(h^{1 / 2}\right) .
$$


Proof. Let $h>0$ and let $g_{h}$ be the solution of the Poisson equation $\mathcal{L} g=-f /(2 h)$. Note that by Lemma $4 g_{h}=\sum_{l=1}^{\infty} a_{l, h} \phi_{l}$ where

$$
a_{l, h}=\frac{2 l+1}{2 h l(l+1)}\left\langle\phi_{l}, f\right\rangle_{\pi} .
$$

By Theorem 3. $L g_{h}=2 h \mathcal{L} g_{h}+O\left(h^{1 / 2}\right)=-f+O\left(h^{1 / 2}\right)$. Note that the error in the above expression is of lower order than that in the theorem because the right hand side in the Poisson equation contains a factor of $h^{-1}$. Next observe that

$$
\begin{aligned}
\left\langle P f, g_{h}\right\rangle_{\pi} & =\left\langle f, P g_{h}\right\rangle_{\pi} \\
& =\left\langle f, g_{h}\right\rangle_{\pi}+2 h\left\langle f, \mathcal{L} g_{h}\right\rangle_{\pi}+O\left(h^{1 / 2}\right) \\
& =\frac{1}{2 h} \sum_{l=1}^{\infty} \frac{2 l+1}{l(l+1)}\left\langle\phi_{l}, f\right\rangle_{\pi}^{2}-\langle f, f\rangle_{\pi}+O\left(h^{1 / 2}\right) .
\end{aligned}
$$

Using the expression above, along with the formula for $\sigma_{f, h}^{2}$ given in 12 , the result then follows.

\section{AnAlysis of the GalERKin METHOD}

In this section, we conclude with an analysis of the Galerkin method introduced in Subsection 2.6. including a proof of Theorem 5. We begin with a result on the decay rates of Legendre series truncation which will be useful. It is taken from Theorem 2.2 from [21], restated slightly here to fit our notation and context.

We will give estimates in terms of the following weighted semi-norm, defined on the space of functions $u:(-1,1) \rightarrow \mathbb{R}$ such that the following integral is defined:

$$
\|u\|_{w}=\int_{-1}^{1} \frac{\left|u^{\prime}(x)\right|}{\left(1-x^{2}\right)^{\frac{1}{4}}} d x .
$$

Theorem 9 (Adapted from Theorem 2.2 in [21]). Let $m \geq 1$, and let $u:(-1,1) \rightarrow \mathbb{R}$ be $a$ function such that $u, u^{\prime}, \ldots, u^{(m-1)}$ are absolutely continuous and the $m$-th derivative $u^{(m)}$ is of bounded variation. Furthermore, assume that $\left\|u^{(m)}\right\|_{w}<\infty$. Let $a_{n}=(2 n+1)\left\langle u, \phi_{n}\right\rangle$ be the sequence of coefficients in the Legendre expansion of $u$ such that $u(x)=\sum_{n=0}^{\infty} a_{n} \phi_{n}(x)$. Then, for $n \geq m+1$,

$$
\left|a_{n}\right| \leq \frac{\left\|u^{(m)}\right\|_{w}}{\sqrt{\pi(2 n-2 m-1)}} \prod_{k=1}^{m} \frac{2}{2 n-2 k+1} .
$$

We are now ready to prove Theorem 5 . We begin by recalling some notation introduced in Subsection 2.6. Let $T_{n}: L_{0}^{2}(X, \pi) \rightarrow R_{n}$ denote the orthogonal projection to the linear span $R_{n}=\left\{\phi_{1}, \ldots, \phi_{n}\right\}$ of the first $n$ non-constant Legendre polynomials. The solution of the finite dimensional linear system $\left(I-T_{n} P\right) x=T_{n} f$, where $f \in L_{0}^{2}(X, \pi)$ is the given observable, will be written as $g_{n}$. Note that $g_{n} \in R_{n}$, and writing $g_{n}=\sum_{j=1}^{n} \alpha_{j} \phi_{j}$, it is straightforward to see that we aim to find a solution $x$ to the system $G x=y$ where

$$
x=\left(\alpha_{1}, \ldots, \alpha_{n}\right)^{\top}, \quad y=\left(\left\langle f, \phi_{1}\right\rangle_{\pi}, \ldots,\left\langle f, \phi_{n}\right\rangle_{\pi}\right)^{\top}, \quad G=\left(\left\langle\phi_{j}, \phi_{i}\right\rangle_{\pi}-\left\langle P \phi_{j}, \phi_{i}\right\rangle_{\pi}\right)_{i, j=1}^{n} .
$$


The solution $g_{n}$ will in turn be used to give the approximation $\sigma_{\mathrm{GM}, n}^{2}:=\left\langle T_{n} f, T_{n} f\right\rangle+2\left\langle T_{n} P f, g_{n}\right\rangle$ of the diffusivity $\sigma_{f}^{2}$.

Proof of Theorem 5. Throughout the proof, we take $P$ to be the restriction of the Markov operator to the space $L_{0}^{2}(X, \pi)$ so that $\|P\|<1$, where $\|\cdot\|$ denotes $L^{2}$-operator norm. Observe that from the definition of $\sigma_{\mathrm{GM}, n}^{2}$,

$$
\left|\sigma_{f}^{2}-\sigma_{\mathrm{GM}, n}^{2}\right| \leq\langle f, f\rangle_{\pi}-\left\langle T_{n} f, T_{n} f\right\rangle_{\pi}|+2|\langle P f, g\rangle_{\pi}-\left\langle T_{n} P f, g_{n}\right\rangle_{\pi} \mid .
$$

Our aim is to show that the two terms of the right hand side above are bounded by a common factor in terms of $n$, which we will then show decays as in the statement of the theorem. For the first term on the right hand side of $(15)$, we see that

$$
\begin{aligned}
\langle f, f\rangle_{\pi}-\left\langle T_{n} f, T_{n} f\right\rangle_{\pi} & =\|f\|_{\pi}^{2}-\left\|T_{n} f\right\|_{\pi}^{2} \\
& \leq 2\|f\|_{\pi}\left(\|f\|_{\pi}-\left\|T_{n} f\right\|_{\pi}\right) \\
& \leq 2\|f\|_{\pi}\left\|f-T_{n} f\right\|_{\pi},
\end{aligned}
$$

and for the second term,

$$
\begin{aligned}
\left|\langle P f, g\rangle_{\pi}-\left\langle T_{n} P f, g_{n}\right\rangle_{\pi}\right| & =\left|\int_{X}\left[P f(x) g(x)-T_{n} P f(x) g_{n}(x)\right] \pi(d x)\right| \\
& \leq \int_{X}\left|P f(x)\left(g(x)-g_{n}(x)\right)\right| \pi(d x)+\int_{X}\left|g_{n}(x)\left(P f(x)-T_{n} P f(x)\right)\right| \pi(d x) \\
& \leq\|P f\|_{\pi}\left\|g-g_{n}\right\|_{\pi}+\left\|g_{n}\right\|_{\pi}\left\|P f-T_{n} P f\right\|_{\pi} \\
& \leq\|P f\|_{\pi}\left\|\left(I-T_{n} P\right)^{-1}\right\|\left\|g-T_{n} g\right\|_{\pi}+\left\|g_{n}\right\|_{\pi}\left\|P f-T_{n} P f\right\|_{\pi},
\end{aligned}
$$

where in the last step we have used the fact that $g-g_{n}=\left(I-T_{n} P\right)^{-1}\left(g-T_{n} g\right)$.

It is straightforward to see that $T_{n} P=P T_{n}$. Indeed, for any function $f \in L_{0}^{2}(X, \pi)$, with Legendre expansion given by $f=\sum_{k=1}^{\infty} a_{k} \phi_{k}$, we have that $P f(x)=\sum_{k=1}^{\infty} a_{k} P \phi(x)$. Applying $T_{n}$ to both sides of this equation, we get that $T_{n} P f(x)=\sum_{k=1}^{n} a_{k} P \phi(x)=P T_{n} f(x)$. It now follows that

$$
\left\|P f-T_{n} P f\right\|_{\pi}=\left\|P f-P T_{n} f\right\|_{\pi} \leq\|P\|\left\|f-T_{n} f\right\|_{\pi} .
$$

Moreover, a similar argument gives that

$$
\left\|g-T_{n} g\right\|_{\pi} \leq\left\|(I-P)^{-1}\right\|\left\|f-T_{n} f\right\|_{\pi} \leq(1-\|P\|)^{-1}\left\|f-T_{n} f\right\|_{\pi} .
$$

Finally, we note that

$$
\left\|\left(I-T_{n} P\right)^{-1}\right\| \leq\left(1-\left\|T_{n} P\right\|\right)^{-1} \leq(1-\|P\|)^{-1},
$$

and consequently,

$$
g_{n}=\left(I-T_{n} P\right)^{-1} T_{n} f \leq(1-\|P\|)^{-1}\|f\|_{\pi} .
$$

We then have for the second term on the right hand side of 15 ,

$$
\left|\langle P f, g\rangle_{\pi}-\left\langle T_{n} P f, g_{n}\right\rangle_{\pi}\right| \leq\|P\|(2-\|P\|)(1-\|P\|)^{-2}\|f\|_{\pi}\left\|f-T_{n} f\right\|_{\pi} .
$$


Applying the estimates in (16) and (17) to 15 and simplifying, we have that

$$
\left|\sigma_{f}^{2}-\sigma_{\mathrm{GM}, n}^{2}\right| \leq 2\|f\|_{\pi}(1-\|P\|)^{-2}\left\|f-T_{n} f\right\|_{\pi} .
$$

It is now evident that the convergence rate will depend on the decay rate of $f$ with it's Legendre series truncation. Observe that

$$
\left\|f-T_{n} f\right\|_{\pi} \leq \sum_{k=n+1}^{\infty} a_{k}\left\|\phi_{k}\right\|_{\pi}=\sum_{k=n+1}^{\infty}(2 k+1)^{-1 / 2} a_{k},
$$

where $a_{k}=(2 k+1)\left|\left\langle f, \phi_{k}\right\rangle_{\pi}\right|$. Using Theorem 9 with $m=1$ we get

$$
\left|a_{k}\right| \leq \frac{2\left\|f^{\prime}\right\|_{w}}{\sqrt{\pi}(2 k-1) \sqrt{2 k-3}} .
$$

Thus

$$
\left\|f-T_{n} f\right\|_{\pi} \leq \frac{2 s_{n}\left\|f^{\prime}\right\|_{\mathrm{w}}}{\sqrt{\pi}}
$$

where

$$
s_{n}:=\sum_{k=n+1}^{\infty} \frac{1}{(2 k-1) \sqrt{2 k+1} \sqrt{2 k-3}} .
$$

Further, for $n>2$ we have

$$
\begin{aligned}
s_{n} & <\sum_{i=n+1}^{\infty} \frac{1}{(2 i-3)^{2}} \\
& \leq \int_{n}^{\infty} \frac{d x}{(2 x-3)^{2}} \\
& =\frac{1}{4 n-6} .
\end{aligned}
$$

The result now follows by applying these estimates to (18).

\section{REFERENCES}

[1] J. M. Anderson et al. Isothermal mass flow measurements in microfabricated rectangular channels over a very wide Knudsen range. J. Micromech. Microeng., 24 (2014), 055013

[2] K. Atkinson and A. Bogomolny The discrete Galerkin method for integral equations Mathematics of computation, 48:595-616 (1987)

[3] O. Angel, K. Burdzy, and S. Sheffield. Deterministic approximations of random reflectors. Trans. Amer. Math. Soc., 365(12):6367-6383, 2013.

[4] G. Arya, H.-C. Chang, and E. J. Maginn. Knudsen diffusivity of a hard sphere in a rough slit pore. Phys. Rev. Lett., 91:026102, Jul 2003.

[5] C. Barnes, K. Burdzy, and C.-E. Gauthier. Billiards with Markovian reflection laws. Electron. J. Probab., 24:Paper No. 147, 32, 2019. 
[6] N. Chernov and R. Markarian. Chaotic billiards, volume 127 of Mathematical Surveys and Monographs. American Mathematical Society, Providence, RI, 2006.

[7] T. Chumley, R. Feres, and H.-K. Zhang. Diffusivity in multiple scattering systems. Trans. Amer. Math. Soc., 368(1):109-148, 2016.

[8] F. Comets, S. Popov, G. M. Schütz, and M. Vachkovskaia. Billiards in a general domain with random reflections. Arch. Ration. Mech. Anal., 191(3):497-537, 2009.

[9] S. Cook and R. Feres. Random billiards with wall temperature and associated Markov chains. Nonlinearity, 25(9):2503-2541, 2012.

[10] S. N. Evans. Stochastic billiards on general tables. Ann. Appl. Probab., 11(2):419-437, 2001.

[11] R. Feres. Random walks derived from billiards. In Dynamics, ergodic theory, and geometry, volume 54 of Math. Sci. Res. Inst. Publ., pages 179-222. Cambridge Univ. Press, Cambridge, 2007.

[12] R. Feres, J. Ng, and H.-K. Zhang. Multiple scattering in random mechanical systems and diffusion approximation. Comm. Math. Phys., 323(2):713-745, 2013.

[13] R. Feres and G. Yablonsky. Knudsen's cosine law and random billiards. Chemical engineering science, 59(7):1541-1556, 2004.

[14] R. Feres and H.-K. Zhang. Spectral gap for a class of random billiards. Comm. Math. Phys., 313(2):479-515, 2012.

[15] K. Khanin and T. Yarmola. Ergodic properties of random billiards driven by thermostats. Comm. Math. Phys., 320(1):121-147, 2013.

[16] C. Kipnis and S. R. S. Varadhan. Central limit theorem for additive functionals of reversible Markov processes and applications to simple exclusions. Comm. Math. Phys., 104(1):1-19, 1986.

[17] L. Marino. Experiments on rarefied gas flows through tubes. Microfluidics Nanofluidics (2009) 6:109-119

[18] P. Perrier, I. A. Graur, T. Ewart, and J. G. Méolans Mass flow rate measurements in microtubes: From hydrodynamic to near free molecular regime. Physics of Fluids 23, 042004 (2011)

[19] G. O. Roberts and J. S. Rosenthal. Geometric ergodicity and hybrid Markov chains. Electron. Comm. Probab., 2:no. 2, 13-25, 1997.

[20] S. Varoutis, S. Naris, V. Hauer, C. Day, and D. Valougeorgis. Computational and experimental study of gas flows through long channels of various cross sections in the whole range of the knudsen number. Journal of Vacuum Science \& Technology A, 27(1):89-100, 2009.

[21] H. Wang. A new and sharper bound for legendre expansion of differentiable functions. Applied Mathematics Letters, 85:95-102, 2018. 
[22] J. Weidmann. Linear operators in Hilbert spaces, volume 68 of Graduate Texts in Mathematics. Springer-Verlag, New York-Berlin, 1980. Translated from the German by Joseph Szücs.

[23] H. Yamaguchi, Y. Matsuda, and T. Niimi. Tangential Momentum Accommodation Coefficient measurements for various materials and gas species. Journal of Physics: Conference Series 362 (2012) 012035 1st European Conference on Gas Micro Flows (GasMems 2012) 